\title{
Atwood ratio dependence of Richtmyer-Meshkov flows under reshock conditions using large-eddy simulations
}

\author{
M. LOMBARDINI†, D. J. HILL, D. I. PULLIN \\ AND D. I. MEIRON
}

Graduate Aeronautical Laboratories, California Institute of Technology, Pasadena, CA 91125, USA

(Received 11 January 2010; revised 18 September 2010; accepted 8 October 2010;

first published online 1 February 2011)

We study the shock-driven turbulent mixing that occurs when a perturbed planar density interface is impacted by a planar shock wave of moderate strength and subsequently reshocked. The present work is a systematic study of the influence of the relative molecular weights of the gases in the form of the initial Atwood ratio $A$. We investigate the cases $A= \pm 0.21, \pm 0.67$ and \pm 0.87 that correspond to the realistic gas combinations air- $\mathrm{CO}_{2}$, air- $-\mathrm{SF}_{6}$ and $\mathrm{H}_{2}$-air. A canonical, threedimensional numerical experiment, using the large-eddy simulation technique with an explicit subgrid model, reproduces the interaction within a shock tube with an endwall where the incident shock Mach number is $\sim 1.5$ and the initial interface perturbation has a fixed dominant wavelength and a fixed amplitude-to-wavelength ratio $\sim 0.1$. For positive Atwood configurations, the reshock is followed by secondary waves in the form of alternate expansion and compression waves travelling between the endwall and the mixing zone. These reverberations are shown to intensify turbulent kinetic energy and dissipation across the mixing zone. In contrast, negative Atwood number configurations produce multiple secondary reshocks following the primary reshock, and their effect on the mixing region is less pronounced. As the magnitude of $A$ is increased, the mixing zone tends to evolve less symmetrically. The mixing zone growth rate following the primary reshock approaches a linear evolution prior to the secondary wave interactions. When considering the full range of examined Atwood numbers, measurements of this growth rate do not agree well with predictions of existing analytic reshock models such as the model by Mikaelian (Physica D, vol. 36, 1989, p. 343). Accordingly, we propose an empirical formula and also a semianalytical, impulsive model based on a diffuse-interface approach to describe the $A$-dependence of the post-reshock growth rate.

Key words: instability, shock waves, turbulent mixing

\section{Introduction}

When a perturbed density interface separating two fluids is impacted by a shock wave, vorticity is deposited at the interface by means of baroclinic torque, due to the mis-alignment of the pressure gradient $\nabla p$ across the shock and the local density gradient $\nabla \rho$ at the interface. Perturbations grow and eventually develop complex 


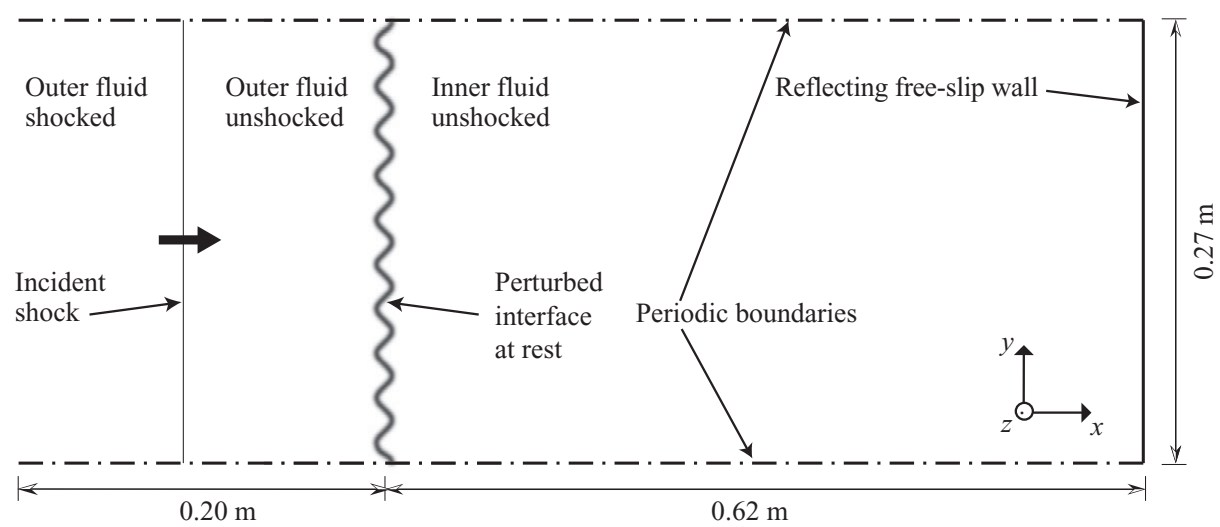

FIGURE 1. Geometry of the simulation domain before an incident shock impacts a single-mode perturbed density interface, separating two quiescent fluids of different densities. Note that the domain is squared in the $(y, z)$-cross-section.

structure, forming a mixing zone of interpenetrating light and heavy fluids. This class of instabilities, also known as the Richtmyer-Meshkov instability (RMI), is often thought of as an impulsive or shock-induced version of the continuously driven Rayleigh-Taylor instability (RTI), which occurs at accelerated density-stratified interfaces. The RMI derives its name from the numerical and analytical predictions of Richtmyer (1960), subsequently confirmed by the shock tube experimental results of Meshkov (1969). In the RTI case, the interface becomes unstable if the density gradient is opposite in its direction to the acceleration, i.e. $\nabla p \cdot \nabla \rho<0$ (e.g. heavy fluid lying on top of a lighter fluid in a gravitational field). However, in the RMI case, perturbation growth will result whether the incident shock wave propagates from a light to a heavy gas $(\nabla p \cdot \nabla \rho<0)$ or from a heavy to a light gas $(\nabla p \cdot \nabla \rho>0$, in which case phase reversal precedes the growth), and such shock-interface interactions need to be distinguished. In shock tube experiments with an endwall (e.g. as described here in figure 1), the initial shock-contact interaction and the reshock will be of alternate classifications as the shock reverses its direction of propagation when it reflects off the endwall, as will be described here.

The physics of reshock environments involving the RTI and RMI is under investigation due to its relevance to applications spanning a wide range of scales, such as supernovae dynamics and inertial confinement fusion (ICF). In these geometrically converging configurations, the incident imploding wave bounces off the centre towards the initially accelerated density stratifications. The subsequent reshock enhances the mixing process between the different density layers. This was confirmed by the observations of Supernova 1987A (Arnett et al. 1989), which indicate a significant amount of Richtmyer-Meshkov mixing between the stratified outer gas layers following the core collapse (Burrows, Hayes \& Fryxell 1995). At smaller scales, the RMI represents a considerable obstacle to achieving a productive fusion reaction by limiting the compression of the deuterium-tritium fuel (Lindl, McCrory \& Campbell 1992; Taccetti et al. 2005). The majority of simulations of ICF capsule implosions are still performed in one and two dimensions, as the computational resources required to fully resolve the range of spatial and temporal scales in three-dimensional turbulent flows are still beyond the reach of available computing facilities. To reduce the computational costs, Lombardini, Deiterding \& Pullin (2008) have used the techniques of large-eddy simulation (LES) within an adaptive mesh refinement (AMR) framework to perform 
three-dimensional computations of shock-induced mixing occurring at cylindrically converging light-heavy interfaces. These canonical simulations constitute a first step in reliably reproducing the compressible turbulent mixing in highly compressible environments inherent to converging geometries. On a more conceptual note, the turbulent intensification following the reshock raises the issue of the mixing transition in shock-driven flows (Dimotakis 2000; Tomkins et al. 2008).

Among fundamental studies on reshock systems, Brouillette \& Sturtevant (1989, 1993 , 1994) reported on the growth induced by successive shock waves for air-air $(A \approx 0)$, the light-heavy configurations air- $\mathrm{CO}_{2}(A \approx 0.20)$, air- $\mathrm{R} 22(A \approx 0.50)$, air$\mathrm{SF}_{6}(A \approx 0.67)$, and the heavy-light configuration air-He $(A \approx-0.76)$, and examined the shock tube experiments involving initially discontinuous or continuous interfaces. Disagreements with original experiments by Andronov et al. (1976) and Zaitsev et al. (1985) have been attributed to differences between the experimental set-ups, as well as to the existence of a wall vortex on the sidewalls caused by the interaction of the reflected shock with the mixing zone within the boundary layer. Thereafter, Houas \& Chemouni (1996) presented experimental results essentially on heavy-light discontinuous interfaces separating $\mathrm{CO}_{2}$ with different noble gases ( $A$ approximately ranging from -0.76 to -0.14 ) impacted by rather strong incident shock waves, concluding that their thin membrane significantly affected the measurement of the mixing-layer width.

Using results from early RTI experiments (Read 1984), RTI and RMI simulations (Youngs 1984), and theory relating to RTI and RMI (Canuto \& Goldman 1985), Mikaelian (1989) produced a theoretical RMI model predicting a growth linear in time for the phase following the reshock, neglecting molecular dissipation effects and assuming no dependence on the initial conditions. Other analytical or semi-analytical models (Brouillette \& Sturtevant 1994; Charakhchyan 2001) also predict a linear growth in time. More recently, Mikaelian (2009) investigated the use of a generalized Layzer model in two different shock tube configurations: with endwall (reshock produced) and with 'free' boundary (rarefaction reflected from a membrane). The performance of the Layzer model and hydrocode simulations was compared in both configurations (focusing essentially on the nonlinear evolution of bubble fronts), and remains to be verified experimentally.

The interaction of an expansion wave with a growing mixing layer generally occurs in heavy-to-light reshock configurations (i.e. initial light-to-heavy shock interaction, as depicted in more detail later in figure 2). Indeed, the reshock produces a reflected expansion travelling into the heavy fluid, reflecting from the end boundary and interacting with the mixing layer. In one of the first comprehensive studies of the role of this expansion wave on the mixing-layer growth, Hill, Pantano \& Pullin (2006) reproduced numerically the shock tube experiments of Vetter \& Sturtevant (1995) using three-dimensional LES. In that study, the target experiment consists of a planar shock of moderate Mach number, ranging from 1.24 to 1.98 , interacting with a co-planar perturbed density interface formed by the contact of air and $\mathrm{SF}_{6}$ (oriented so that the first shock interaction is light-to-heavy), with an Atwood ratio $A \approx-0.67$ (see exact definition of $A$ in the following section). An examination of the turbulent kinetic energy (TKE) indicated that the first expansion wave following the reshock plays a major role in driving the evolution of the mixing zone. In addition to presenting the evolution of the mixing-layer amplitude, Hill et al. (2006) also described the flow evolution through the representation of various turbulent statistics extracted across the mixing layer, such as TKE, turbulent dissipation, velocity and scalar power spectra. Another contribution is the computational work by Schilling, 
Latini \& Don (2007) on two-dimensional RMI followed by reshock, using shockcapturing weighted essentially non-oscillatory (WENO) schemes of formally higher order (Latini, Schilling \& Don 2007) without an explicit dissipation or a model of the unresolved scales. These simulations reproduce some of the observations of Collins \& Jacobs (2002) obtained from membrane-free shock tube experiments on the RMI at a light-heavy (air (acetone) $\mathrm{SF}_{6}, A \approx 0.60$ ) single-mode interface impacted by a Mach 1.21 shock. Schilling et al. (2007) compare the simulated mixing-layer amplitude with existing reshock models evaluated at the same Atwood ratio (Mikaelian 1989; Brouillette \& Sturtevant 1994; Charakhchyan 2001) during the post-reshock/preexpansion phase. Lacking complications present in experimental studies, both Schilling et al. (2007) and Hill et al. (2006) were able to examine the flow evolution over a wider time period following reshock than is customarily reported. In particular, we summarize here conclusions by Schilling et al. (2007) on the effect of the expansion wave on the growth of the two-dimensional mixing layer: the expansion wave has a modest effect on the mixing kinetic energy, enstrophy and density fluctuations, leads to an increase of the spanwise velocity fluctuations compared with the streamwise counterpart, contributes to the late-time isotropization of the velocity field, and is responsible for the symmetry breaking and stretching of the mixing layer.

Very recent reshock experiments have been reported using air- $\mathrm{SF}_{6}$ with a randomly perturbed interface impacted by low-to-moderate Mach number shock, ranging from about 1.1 to 1.5 (Leinov et al. 2009) and configured so as to produce a light-to-heavy interaction. Leinov et al. (2009) demonstrate, for their experimental configuration, that the post-reshock growth amplitude is insensitive to the initial amplitude of the interface perturbation for that range of Mach number (for similar observations, see for example Vetter \& Sturtevant 1995). These conclusions are drawn for a rather restrictive span of incident shock strengths and are limited to a narrow time window ending before the passage of the expansion wave.

In general, the RMI can be studied in a three-parameter space of initial conditions: the initial shape of the perturbed interface, the incident shock strength and the gas combination forming the density interface. Although the effect of initial perturbations (Erez et al. 2000; Dimonte 2004; Greenough \& Burke 2004; Gupta et al. 2007) and incident Mach number (Samtaney \& Zabusky 1993; Holmes et al. 1999; Orlicz et al. 2009) has been the subject of extensive research, the influence of the Atwood ratio has been little studied in the literature. Previous work has mainly focused on the nonlinear evolution of the two unstable fingering structure fronts in such flows, known as spikes and bubbles, for initially sharp multi-mode interfaces (when the intrinsic interface thickness is small compared with the perturbation amplitude), e.g. as described by Alon et al. (1995), Dimonte \& Schneider (2000) and Oron et al. (2001). Growth-reduction mechanisms that account for diffusion at the interface have also been proposed to implicitly describe the $A$-dependence, in the presence of reshock (Brouillette \& Sturtevant 1994, for light-heavy configurations) or for single-shock interactions (Motl et al. 2009, also for light-heavy configurations, such as mixtures of $\mathrm{He}$ and $\operatorname{Ar}(A \approx 0.29), \mathrm{Ne}-\mathrm{SF}_{6}(A \approx 0.68)$ and $\left.\mathrm{He}-\mathrm{SF}_{6}(A \approx 0.95)\right)$. Samtaney \& Zabusky (1993) have also proposed an analytical density-ratio dependence on the baroclinic circulation initially deposited at a planar light-to-heavy interface. In shockbubble interactions (Giordano \& Burtschell 2006; Niederhaus et al. 2008; Layes, Jourdan \& Houas 2009, among recent studies that consider various gas pairings), the refracted portion of the incident wave results in a complex wave distortion process in the interior of the bubble, particularly at high density ratios. The reverberation of these reflected and/or diffracted waves is analogous to Richtmyer-Meshkov growth 
after reshock. In these three studies, the main configurations considered were lightheavy air-Kr $(A \approx 0.49)$ and heavy-light air-He $(A \approx-0.76)$, where the bubbles were processed by weak-to-moderate incident shock waves in the experiments by Giordano \& Burtschell (2006) and Layes et al. (2009), and even stronger shocks in the computations by Niederhaus et al. (2008).

We present a systematic study of the $A$-dependence of shock-driven mixing under reshock conditions, employing both numerical and analytical strategies. Our numerical computations provide a detailed description of the different stages leading eventually to the turbulent mixing, while the analysis considers the impulsive acceleration of a diffuse interface as a model for the reshock of a turbulent mixing layer.

The present work reports on detailed numerical simulations of a canonical problem for the three-dimensional planar RMI with reshock, for three light-heavy configurations and their heavy-light counterparts, producing six in total, while the initial Mach number and shape of the interface are fixed. Twelve additional simulations were performed with two alternative, but similar, initial interface shapes so that the sensitivity of the results could be explored. After discussing the initial and boundary conditions in $\S 2$, we briefly describe, in $\S 3$, the relevant filtered NavierStokes equations and the explicit subgrid-scale model used in the simulations, as well as the numerical strategy employed. A description of the flow is given in $\S 4$, followed by a discussion on the reshock growth rate in $\S 5$.

\section{Flow configuration}

In this study, the gas combinations are varied to achieve different Atwood ratios, but in each case the gases are assumed to be initially temperature-matched. Figure 1 shows the canonical experiment, reading from left to right, shocked 'outer' gas, the shock, unshocked outer gas, the contact, the 'inner' gas and the endwall. As a point of terminology, configurations are described by the geometry and relative densities of the gases. In a 'heavy-light' configuration, the density of the outer gas is greater than that of the inner gas, and such an experiment will first produce a 'heavy-tolight' interaction as the shock interacts with the contact, and then a 'light-to-heavy' interaction during reshock. We define the Atwood number $A$ in this geometric context in terms of the pre-shock states of the gases as

$$
A=\frac{\rho_{i}-\rho_{o}}{\rho_{i}+\rho_{o}}
$$

where $\rho_{o}$ and $\rho_{i}$ are the initial densities of the outer and inner gases, respectively.

\subsection{Simulation domain}

The simulation geometry consists of a square-tube configuration with periodic boundary conditions in the two cross-directions $y$ and $z$. The shock tube has crossdimensions $L_{y} \times L_{z}$ of $0.27 \mathrm{~m} \times 0.27 \mathrm{~m}$ and down-tube length of $0.82 \mathrm{~m}$. As explained in $\S 3$, the numerical method is applied within an AMR framework as described by Pantano et al. (2007), utilizing Cartesian grids and cubic cells. The finest resolution $\left(\Delta x=L_{y} / 128\right)$ is applied uniformly in the mixing region. This resolution was found to be sufficient by Hill et al. (2006) for similar problems. Details on the flow geometry and computation are listed in table 1.

The incident shock travels towards the wall from the left to the right in the direction of increasing $x$ and impacts, at $t=0^{+}$, the interface positioned at $x=0$. Reshock then occurs after the transmitted shock reflects off the endwall and returns to the left. The 


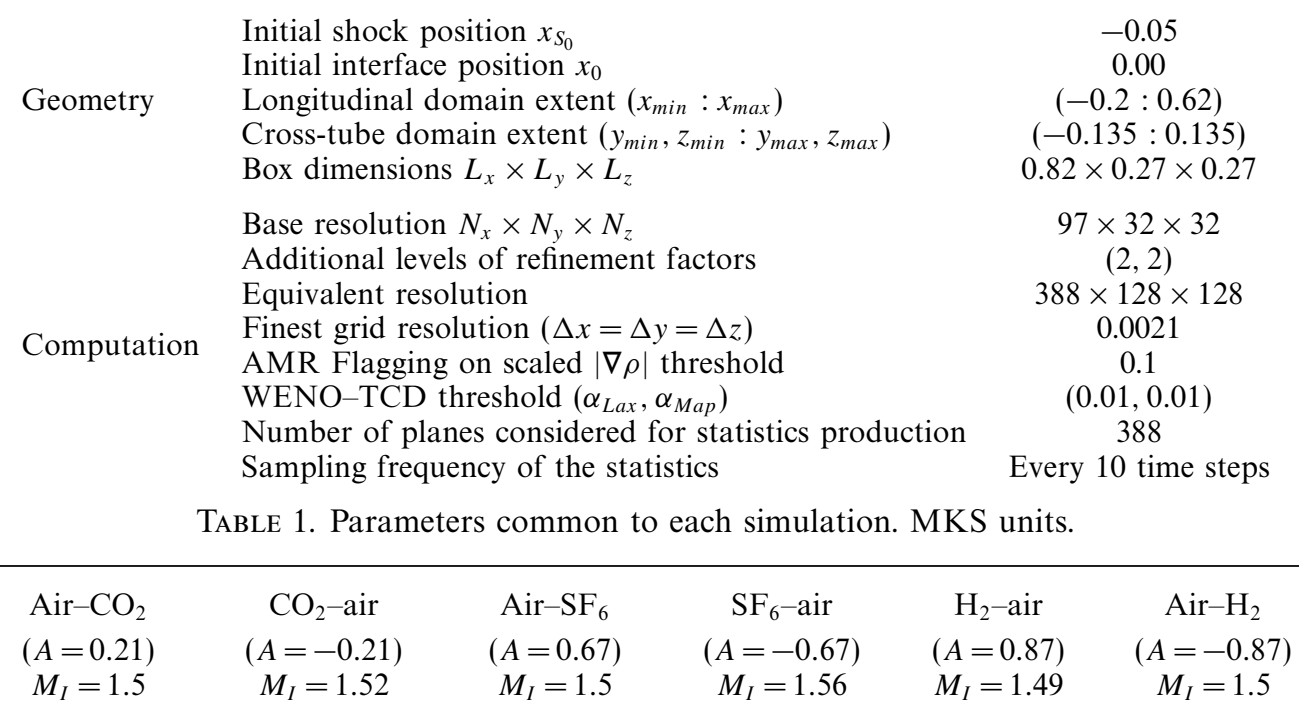

TABLE 2. Gas combinations studied and the corresponding incident Mach numbers considered.

shock then exits the computational domain and the simulation continues into the later phases of the mixing zone evolution.

\subsection{Initial conditions}

We essentially explore a one-dimensional parameter space in which the initial Atwood ratio is varied in both sign and magnitude, as summarized in table 2. For any gas combinations, the temperature $T_{o}$ and pressure $p_{o}$ of unshocked fluid to the left of the interface (the outer fluid) are chosen to be the same: $p_{o}=23 \mathrm{kPa}$ and $T_{o}=286 \mathrm{~K}$ (cf. Vetter \& Sturtevant 1995). Initially, the temperature and pressure are taken to be uniform in the unshocked gases, while the density varies continuously across the twogas interface, as observed experimentally. From $T_{o}$ and $p_{o}$, we compute the density $\rho_{o}$ and sound speed $c_{o}$. From $A$ and $\rho_{o}$, it is straightforward to deduce $\rho_{i}$, the density of the unshocked fluid to the right of the interface (the inner fluid).

In each experiment, air is used in conjunction with one of three gases: $\mathrm{CO}_{2}, \mathrm{SF}_{6}$ and $\mathrm{H}_{2}$. For each gas combination, two configurations are examined: with air as the inner gas, and with air as the outer gas. No attempt was made to incorporate any real-gas effects, such as relaxation in the case of $\mathrm{CO}_{2}$, as such effects are assumed to be negligible. Instead, all gases were modelled as ideal with the physical properties found in table 3 .

In discussing the gas configurations, we follow the convention of listing first the outer gas and then the inner gas. For example, 'AirH ${ }_{2}$ ' or air- $\mathrm{H}_{2}$ indicates a simulation in which the shock is initialized in air, heading towards the contact with the lighter gas $\mathrm{H}_{2}$.

\subsubsection{The initial contact}

We define by $x-x_{I}(y, z)=0$ the surface where light and heavy fluids have equal mass fractions. The initial mass fraction takes the form of a hyperbolic tangent profile 


\begin{tabular}{lcrrc} 
Property & Air & \multicolumn{1}{c}{$\mathrm{SF}_{6}$} & $\mathrm{CO}_{2}$ & $\mathrm{H}_{2}$ \\
Molecular mass $m_{\alpha}\left(\mathrm{kg} \mathrm{kmol}^{-1}\right)$ & 28.83 & 146.07 & 44.01 & 2.02 \\
Atwood ratio with air $A$ & 0.0 & 0.67 & 0.21 & -0.87 \\
Ratio of specific heats $\gamma_{\alpha}$ & 1.40 & 1.09 & 1.29 & 1.41 \\
Density $\rho_{\alpha}\left(\mathrm{kg} \mathrm{m}^{-3}\right)$ & 1.18 & 5.97 & 1.80 & 0.082 \\
Kinematic viscosity $v_{\alpha}\left(10^{-6} \mathrm{~m}^{2} \mathrm{~s}^{-1}\right)$ & 15.7 & 2.47 & 8.35 & 109.1 \\
Prandtl number & 0.71 & 0.90 & 0.76 & 0.69 \\
Diffusion coefficient in air $D_{\alpha}\left(10^{-6} \mathrm{~m}^{2} \mathrm{~s}^{-1}\right)$ & 20.4 & 9.7 & 16.5 & 61.0
\end{tabular}

TABle 3. Gas properties of air, $\mathrm{SF}_{6}, \mathrm{CO}_{2}$ and $\mathrm{H}_{2}$ at $25^{\circ} \mathrm{C}$ and $1 \mathrm{~atm}$.

centred at $x_{I}(y, z)$ and with a characteristic thickness $L_{0}=0.01 \mathrm{~m}$. A symmetrybreaking perturbation containing various modes around the dominant wavenumber $k_{0}=2 \pi / \lambda_{0}, \lambda_{0}=0.027 \mathrm{~m}$ (10 periods per shock tube width), is set up in the $y$ - and $z$-directions. It can be represented by a wavepacket of modes with random phase and whose power spectrum $E\left(k_{y}, k_{z}\right)$, with $k_{y}$ and $k_{z}$ being wavenumbers in both transverse directions, has the following annular Gaussian profile (Dimonte 2004; Dimonte, Ramaprabhu \& Andrews 2007) centred at the wavenumber $k_{0}$ and with variance $\sigma$ :

$$
E\left(k_{r}\right)=\frac{\mathscr{E}_{0}}{(2 \pi)^{3 / 2} k_{0} \sigma} \exp \left[-\frac{\left(k_{r}-k_{0}\right)^{2}}{2 \sigma^{2}}\right],
$$

where $k_{r}=\sqrt{k_{y}^{2}+k_{z}^{2}}$ defines the radial wavenumber. As $\sigma \rightarrow 0$, the power spectrum truly represents the single mode $k_{0}$ with random orientations. The total energy $\int_{0}^{\infty} 2 \pi k_{r} E\left(k_{r}\right) \mathrm{d} k_{r}$ is equal to $\mathscr{E}_{0}$ and is directly related to the square of the signal's $L^{2}$-norm. The constant $\mathscr{E}_{0}$ is taken such that the present interface perturbation and the equivalent two-dimensional single-mode perturbation, with wavenumber $k_{0}$ in the $y$-and $z$-directions and maximum amplitude $\eta_{0}=0.0025 \mathrm{~m}$, have the same initial 'mixing-layer' width $\delta_{0}$ defined by

$$
\delta_{0}=4 \int_{x_{\min }}^{x_{\max }}[1-\langle\psi\rangle(x ; 0)]\langle\psi\rangle(x ; 0) \mathrm{d} x,
$$

where $\langle\psi\rangle(x ; 0)$ is the $y z$-plane average of the mass fraction $\psi(x, y, z ; t)$ at $t=0$ (see $\S \S 3.7$ and 4.2 for general definitions). In practice, $\sigma$ is taken to be of the order of $k_{0} / 5$ such that the highest wavenumbers are resolved. The perturbation can finally be described in the real space $(y, z)$ by

$$
x_{I}(y, z)=\eta_{0} f(y, z),
$$

where $f(y, z)$ is the normalized function derived from the radial power spectrum $E(k)$. The amplitude $\eta_{0} \sim 0.1 \lambda_{0}$ has been chosen large enough that the perturbation is fully resolved during the incident shock refraction process.

\subsubsection{Incident Mach number}

For the light-heavy (air- $\mathrm{CO}_{2}$ and air $-\mathrm{SF}_{6}$ ) and the heavy-light (air- $\mathrm{H}_{2}$ ) cases, we consider an incident shock travelling in air with a Mach number $M_{I}=1.5$. For this shock strength, the length of the shock tube was chosen to observe the initial growth and saturation before reshock, and to keep the mixing zone away from the endwall (Vetter \& Sturtevant 1995; Hill et al. 2006). This is done to avoid 
complications with boundary-layer interactions (Brouillette \& Sturtevant 1993), which are not modelled here.

For the $\mathrm{CO}_{2}$-air, $\mathrm{SF}_{6}$-air and $\mathrm{H}_{2}$-air cases, the incident Mach numbers are specifically chosen so that the initial growth (as predicted by linear theory) matches their respective opposite Atwood ratio cases in the single-mode case. This can be viewed as matching the initial baroclinic torque at the contact or the intensity of the initial shock-contact interaction. Recall that Richtmyer's impulsive model for the growth rate of a single mode (initial perturbation amplitude $\eta_{0}$ and wavenumber $k$, Atwood ratio $A$ ) impacted by a shock is $k \eta_{0}^{+}\left|A^{+}\right| \Delta u_{x}$, where the superscript ' + ' refers to the post-shock state and $\Delta u_{x}$ is the impulse in velocity gained by the interface in the direction of the shock propagation, which is the $x$-direction (Richtmyer 1960). For small incident Mach numbers, initial and post-shock values for the perturbation amplitude and Atwood ratio have comparable magnitude. This model gives a simple estimate of the intensity of the shock interaction as it can be related to the deposition of baroclinic torque during the shock passage as the baroclinic torque is essentially proportional to the relative gradient of density across the interface, i.e. $A$, and the jump of pressure across the shock, which can be easily related to $\Delta u_{x}$. The initial perturbation being fixed in amplitude $\eta_{0}$ and effective wavenumber for the threedimensional perturbation $k=\sqrt{k_{0_{x}}^{2}+k_{0_{y}}^{2}}=\sqrt{2} k_{0}$, we need to match $\Delta u_{x}$. Table 2 enumerates the six runs representing our six different initial Atwood ratios at suitable incident Mach numbers.

\section{Computational approach}

\subsection{Conservative formulation for multicomponent flows}

We consider non-reacting multicomponent flows. Dissociation and ionization effects occurring at the molecular level are neglected since we are not in the hypersonic regime $(\mathrm{Mach}<5)$, and radiative heat transport is ignored because the local temperature differences are supposed to be sufficiently weak. Gravity effects are negligible compared with inertial effects regarding the high velocity of the computed flows. As a result, the conservation of mass, momentum and energy, supplemented with an advection-diffusion equation for $N-1$ species, $N$ being the total number of fluids present, can be written as

$$
\begin{aligned}
& \frac{\partial \rho}{\partial t}+\frac{\partial \rho u_{j}}{\partial x_{j}}=0, \\
& \frac{\partial \rho u_{i}}{\partial t}+\frac{\partial\left(\rho u_{i} u_{j}+p \delta_{i j}\right)}{\partial x_{j}}-\frac{\partial \sigma_{i j}}{\partial x_{j}}=0, \\
& \frac{\partial E}{\partial t}+\frac{\partial(E+p) u_{j}}{\partial x_{j}}-\frac{\partial}{\partial x_{j}}\left(\sigma_{j i} u_{i}-q_{c_{j}}-q_{d_{j}}\right)=0, \\
& \frac{\partial \rho Y_{\alpha}}{\partial t}+\frac{\partial \rho Y_{\alpha} u_{j}}{\partial x_{j}}+\frac{\partial J_{\alpha_{j}}}{\partial x_{j}}=0, \quad \alpha=1, \ldots, N,
\end{aligned}
$$

where the summation convention applies over repeated Roman indices, and where $\rho$ is the density, $u_{j}$ are the velocity components, $p$ is the pressure, and $\sigma_{i j}$ are the viscous stress tensor components, given for Newtonian fluids by

$$
\sigma_{i j}=\mu\left[\left(\frac{\partial u_{i}}{\partial x_{j}}+\frac{\partial u_{j}}{\partial x_{i}}\right)-\frac{2}{3} \frac{\partial u_{k}}{\partial x_{k}} \delta_{i j}\right],
$$


with $\mu$ being the dynamic viscosity. The total energy $E$ for multicomponent flows is

$$
E=\rho \sum_{\alpha=1}^{N} Y_{\alpha} h_{\alpha}-p+\frac{1}{2} \rho u_{i} u_{i}
$$

in which each individual species $\alpha$ has mass fraction $Y_{\alpha}$ and enthalpy $h_{\alpha}$. The conductive heat flux $q_{c_{j}}$ is described by Fourier's law as

$$
q_{c_{j}}=-\kappa \frac{\partial T}{\partial x_{j}}
$$

where $\kappa$ is the thermal conductivity and $T$ is the temperature.

The multicomponent fluid is modelled as a binary mixture of species $\alpha$ and a composite species made of the other materials. Mass diffusion created by temperature gradients, also known as the Soret or thermal-diffusion effect, is neglected. In this approximation, the diffusive mass flux components $J_{\alpha_{j}}$ of the species $\alpha$ are computed using Fick's first law

$$
J_{\alpha_{j}}=-\rho\left(D_{\alpha} \frac{\partial Y_{\alpha}}{\partial x_{j}}-Y_{\alpha} \sum_{\beta=1}^{N} D_{\beta} \frac{\partial Y_{\beta}}{\partial x_{j}}\right),
$$

where $D_{\alpha}$ is the mass diffusion coefficient for species $\alpha$ in the mixture. From (3.5), $\sum_{\alpha=1}^{N} J_{\alpha_{j}}=0$, which ensures that $(3.1 a)$ is recovered when $(3.1 d)$ is summed over all species. The heavy-fluid mass fraction of the $N$ th species can be then obtained after solving $(3.1 d)$ for the $N-1$ other species, using that $\sum_{\alpha=1}^{N} Y_{\alpha}=1$. Because changes in composition of the mixture due to mass diffusion induce changes in internal energy, an interdiffusional enthalpy flux, with components $q_{d_{j}}$ :

$$
q_{d_{j}}=\sum_{\alpha=1}^{N} h_{\alpha} J_{\alpha_{j}}
$$

is present in the right-hand side of the total energy equation (3.1c). It has also been assumed that the relative kinetic energy differences between species are negligible, since the species velocities differ only slightly from the velocity field of the multicomponent fluid.

We incorporated the dependences $\mu\left(Y_{1}, \ldots, Y_{N}, T\right), \kappa\left(Y_{1}, \ldots, Y_{N}, T\right)$ and $D_{\alpha}(T)$, following the mixing rules and pure component transport properties described by Reid, Prausnitz \& Polling (1987). These introduce an additional nonlinearity in the momentum, energy and mass fraction equations.

The system of (3.1)-(3.6) must be completed by an equation of state for the mixture. Each individual species $\alpha$ is assumed to have the properties of an ideal gas, for which the enthalpy is

$$
h_{\alpha}=c_{p_{\alpha}} T
$$

where the specific heat $c_{p_{\alpha}}$ of the pure fluid $\alpha$ is assumed temperature-independent. Thus, the total energy can be reduced to the following form:

$$
E=\rho c_{p} T-p+\frac{1}{2} \rho u_{i} u_{i}=\frac{p}{\gamma-1}+\frac{1}{2} \rho u_{i} u_{i}
$$


The pressure and specific heat ratio of the mixture that appear in the above expressions satisfy

$$
p=\rho \frac{R}{m} T, \quad \gamma=\frac{c_{p}}{c_{p}-R / m},
$$

where $R$ is the universal ideal gas constant, and the specific heat and molecular weight of the mixture are functions of the specific heat and molecular weights of the pure species as

$$
c_{p}=\sum_{\alpha=1}^{N} Y_{\alpha} c_{p_{\alpha}}, \quad \frac{1}{m}=\sum_{\alpha=1}^{N} \frac{Y_{\alpha}}{m_{\alpha}} .
$$

Assuming that all $D_{\alpha}$ are equal, $D_{\alpha} \equiv D$, the diffusive mass flux and interdiffusional enthalpy flux components then reduce to

$$
J_{\alpha_{j}}=-\rho D \frac{\partial Y_{\alpha}}{\partial x_{j}}, \quad q_{d_{j}}=-\rho D T \sum_{\alpha=1}^{N} c_{p_{\alpha}} \frac{\partial Y_{\alpha}}{\partial x_{j}} .
$$

\subsection{Favre-filtered Navier-Stokes equations}

The reshock process produces a large dynamical range of turbulent scales. LES relies on a mathematically well-established formalism where the smaller scales are discarded using a scale high-pass filter to obtain a desired reduction in the range of scales required for numerical simulation. The resolved part $\bar{f}(\boldsymbol{x})$ of a space variable $f(\boldsymbol{x})$ and the Favre-filtered, or density-weighted part, are defined respectively by

$$
\bar{f}(\boldsymbol{x})=\int G_{\Delta_{c}}\left(\boldsymbol{x}-\boldsymbol{x}^{\prime}\right) f\left(\boldsymbol{x}^{\prime}\right) \mathrm{d} \boldsymbol{x}^{\prime}, \quad \widetilde{f}=\frac{\overline{\rho f}}{\bar{\rho}},
$$

where the convolution kernel $G_{\Delta_{c}}$ is associated with an externally specified cutoff scale $\Delta_{c}$. Favre-filtered quantities appear naturally in the filtered equations of motion. Flow features with length scale smaller than $\Delta_{c}$ are identified as 'subgrid' and require modelling.

In practice, the filtering operation can only be performed explicitly from wellresolved fields obtained from experiments or direct numerical simulations (DNS). However, by Favre-filtering of the Navier-Stokes equations (Zhang, Dahlburg \& Dahlburg 1992), transport equations of motion in a conservative form can be formally obtained for the filtered density $\bar{\rho}$, momentum $\bar{\rho} \widetilde{u}_{i}$, total energy $\bar{E}$, and $\bar{\rho} \widetilde{Y}_{\alpha}$ :

$$
\begin{gathered}
\frac{\partial \bar{\rho}}{\partial t}+\frac{\partial \bar{\rho} \widetilde{u}_{j}}{\partial x_{j}}=0, \\
\frac{\partial \bar{\rho} \widetilde{u}_{i}}{\partial t}+\frac{\partial\left(\bar{\rho} \widetilde{u}_{i} \widetilde{u}_{j}+\bar{p} \delta_{i j}\right)}{\partial x_{j}}-\frac{\partial \check{\sigma}_{i j}}{\partial x_{j}}=-\frac{\partial}{\partial x_{j}}\left[\tau_{i j}-\left(\bar{\sigma}_{i j}-\check{\sigma}_{i j}\right)\right], \\
\frac{\partial \bar{E}}{\partial t}+\frac{\partial(\bar{E}+\bar{p}) \widetilde{u}_{j}}{\partial x_{j}}-\frac{\partial}{\partial x_{j}}\left(\check{\sigma}_{j i} \widetilde{u}_{i}-\check{q}_{c_{j}}-\breve{q}_{d_{j}}\right) \\
=-\frac{\partial}{\partial x_{j}}\left[q_{j}^{T}-q_{j}^{T \partial Y}+\frac{\bar{\rho}}{2}\left(\widetilde{u}_{k} \widetilde{u}_{k} u_{j}-\widetilde{u_{k} u_{k}} \widetilde{u}_{j}\right)\right. \\
\left.\quad-\left(\overline{\sigma_{j i} u_{i}}-\check{\sigma}_{j i} \widetilde{u}_{i}\right)+\left(\bar{q}_{c_{j}}-\check{q}_{c_{j}}\right)+\left(\bar{q}_{d_{j}}-\breve{q}_{d_{j}}\right)\right] \\
\frac{\partial \bar{\rho} \widetilde{Y}_{\alpha}}{\partial t}+\frac{\partial \bar{\rho} \widetilde{Y}_{\alpha} \widetilde{u}_{j}}{\partial x_{j}}+\frac{\partial \check{J}_{\alpha_{j}}}{\partial x_{j}}=-\frac{\partial}{\partial x_{j}}\left[q_{j}^{Y_{\alpha}}+\left(\bar{J}_{\alpha_{j}}-\check{J}_{\alpha_{j}}\right)\right], \quad \alpha=1, \ldots, N,
\end{gathered}
$$


where the filtered Newtonian stress tensor $\check{\sigma}_{i j}$, conductive heat flux $\check{q}_{c_{j}}$, interdiffusional enthalpy flux $\breve{q}_{d_{j}}$ and diffusive mass flux $\breve{J}_{\alpha_{j}}$ are given respectively by

$$
\begin{aligned}
& \check{\sigma}_{i j}=\bar{\mu}\left[\left(\frac{\partial \widetilde{u}_{i}}{\partial x_{j}}+\frac{\partial \widetilde{u}_{j}}{\partial x_{i}}\right)-\frac{2}{3} \frac{\partial \widetilde{u}_{k}}{\partial x_{k}} \delta_{i j}\right], \quad \bar{\mu} \equiv \mu\left(\widetilde{Y}_{1}, \ldots, \widetilde{Y}_{N}, \widetilde{T}\right), \\
& \check{q}_{c_{j}}=-\bar{\kappa} \frac{\partial \widetilde{T}}{\partial x_{j}}, \quad \bar{\kappa} \equiv \kappa\left(\widetilde{Y}_{1}, \ldots, \widetilde{Y}_{N}, \widetilde{T}\right), \\
& \check{q}_{d_{j}}=-\bar{\rho} \widetilde{D} \widetilde{T} \sum_{\alpha=1}^{N} c_{p_{\alpha}} \frac{\partial \widetilde{Y}_{\alpha}}{\partial x_{j}}, \quad \widetilde{D} \equiv D(\widetilde{T}), \\
& \check{J}_{\alpha_{j}}=-\bar{\rho} \widetilde{D} \frac{\partial \widetilde{Y}_{\alpha}}{\partial x_{j}}
\end{aligned}
$$

and the subgrid stress tensor $\tau_{i j}$, turbulent temperature flux $q_{j}^{T}$, subgrid interdiffusional enthalpy flux $q_{j}^{T \partial Y}$ and subgrid scalar flux $q_{j}^{Y_{\alpha}}$ are defined respectively as a function of commutation differences of the type $\widetilde{f g}-\widetilde{f} \widetilde{g}$ :

$$
\begin{aligned}
& \tau_{i j}=\bar{\rho}\left(\widetilde{u_{i} u_{j}}-\widetilde{u}_{i} \widetilde{u}_{j}\right), \\
& q_{j}^{T}=\bar{\rho}\left(\widetilde{c_{p} T u_{j}}-\widetilde{c_{p} T} \widetilde{u_{j}}\right) \text {, } \\
& q_{j}^{T \partial Y}=\bar{\rho} \widetilde{D} \sum_{\alpha=1}^{N} c_{p_{\alpha}}\left(T \widetilde{\frac{\partial Y_{\alpha}}{\partial x_{j}}}-\widetilde{T} \frac{\partial \widetilde{Y}_{\alpha}}{\partial x_{j}}\right), \\
& q_{j}^{Y_{\alpha}}=\bar{\rho}\left(\widetilde{Y_{\alpha} u_{j}}-\widetilde{Y}_{\alpha}{\widetilde{u_{j}}}\right) .
\end{aligned}
$$

The last term of (3.13b) and (3.13d) and the last three terms of (3.13c) are essentially due to the temperature dependence of the momentum, heat and mass diffusion coefficients, and will be neglected because they contribute very little to the total budgets for high-Reynolds-number flows. We also assume that the subgrid triple correlation on the right-hand side of (3.13c) is also small compared with the subgrid temperature flux (Kosovic, Pullin \& Samtaney 2002). The modelling of all four subgrid terms (3.15) is the subject of the next paragraph. In practice, the term $q_{j}^{T \partial Y}$ has been neglected since its ratio to $q_{j}^{T}$ is $\sim 1 / R e, R e$ being a typically large Reynolds number characterizing the turbulent activity (see the Appendix).

Finally, $\bar{p}$ and $\widetilde{T}$ still remain to be expressed as a function of the other filtered quantities we are solving for. Filtering (3.8) and using (3.10a) on the one hand, and filtering (3.9a) while using (3.10b), on the other hand, lead to

$$
\begin{aligned}
& \bar{p}=(\widetilde{\gamma}-1)\left[\bar{E}-\frac{1}{2} \widetilde{\rho}_{i} \widetilde{u}_{i}-\frac{1}{2} \tau_{i i}+\widetilde{c}_{p} \sum_{\alpha=1}^{N}\left(\frac{\widetilde{m}}{m_{\alpha}}-\frac{c_{p_{\alpha}}}{\widetilde{c}_{p}}\right) \phi^{T Y_{\alpha}}\right], \\
& \widetilde{T}=\frac{\widetilde{\gamma}}{\bar{\rho} \widetilde{c}_{p}}\left(\bar{E}-\frac{1}{2} \bar{\rho} \widetilde{u}_{i} \widetilde{u}_{i}-\frac{1}{2} \tau_{i i}\right)+\frac{1}{\bar{\rho}} \sum_{\alpha=1}^{N}\left[(\widetilde{\gamma}-1) \frac{\widetilde{m}}{m_{\alpha}}-\widetilde{\gamma} \frac{c_{p_{\alpha}}}{\widetilde{c}_{p}}\right] \phi^{T Y_{\alpha}},
\end{aligned}
$$

where an additional subgrid term to model appears as a correlation between the temperature field and the scalar field $\alpha$ :

$$
\phi^{T Y_{\alpha}}=\bar{\rho}\left(\widetilde{T Y_{\alpha}}-\widetilde{T} \widetilde{Y}_{\alpha}\right),
$$


and where the filtered specific heat, mean molecular weight and specific heat ratio of the mixture are defined as

$$
\widetilde{c}_{p}=\sum_{\alpha=1}^{N} \widetilde{Y}_{\alpha} c_{p_{\alpha}}, \quad \frac{1}{\widetilde{m}}=\sum_{\alpha=1}^{N} \frac{\widetilde{Y}_{\alpha}}{m_{\alpha}}, \quad \tilde{\gamma}=\frac{\widetilde{c}_{p}}{\widetilde{c}_{p}-R / \widetilde{m}} .
$$

These subgrid corrections to the filtered pressure and temperature fields can ultimately modify the total subgrid flux on the right-hand side of the filtered momentum and energy equations.

In the present study, we focus on the passive mixing between two ideal gases ( $\alpha=1$ or 2$)$ of distinct molecular weights. The conservation equations reduce to six equations for the unknown fields $\bar{\rho}, \widetilde{u}_{i}(i=1,2,3), \bar{E}$ and the heavy-fluid mass fraction $\widetilde{Y}_{1}=1-\widetilde{Y}_{2} \equiv \widetilde{\psi}$ representing the local mixture composition between the heavy gas $(\widetilde{\psi}=1)$ and the lighter one $(\widetilde{\psi}=0)$.

\subsection{The stretched-vortex subgrid-scale model}

The stretched-vortex subgrid-scale model by Misra \& Pullin (1997) is based on an explicit structural modelling of small-scale dynamics. The essential subgrid element is modelled by a distribution of stretching vortices that are approximate solutions of the Navier-Stokes equations (Lundgren 1982). This model has been extended to compressible flows (Kosovic et al. 2002), subgrid scalar transport for a constantdensity fluid (Pullin 2000), and here to multicomponent flows (see the Appendix). In particular, an analytical solution for the local winding of the local resolved scalar (or temperature) field by an elemental subgrid vortex is used. The closure of the multicomponent Favre-filtered Navier-Stokes equations (3.13) to (3.18) is achieved by providing the subgrid terms formally defined in (3.15) and (3.17), and explicitly modelled as

$$
\begin{aligned}
\tau_{i j} & =\bar{\rho} \widetilde{k}\left(\delta_{i j}-e_{i}^{v} e_{j}^{v}\right), \\
q_{j}^{T} & =-\bar{\rho} \frac{\Delta_{c}}{2} \widetilde{k}^{1 / 2}\left(\delta_{i j}-e_{i}^{v} e_{j}^{v}\right) \frac{\partial \widetilde{c}_{p} \widetilde{T}}{\partial x_{i}}, \\
q_{j}^{T \partial \psi} & =\bar{\rho} \widetilde{D}\left(c_{p_{1}}-c_{p_{2}}\right) \frac{\Delta_{c}^{2}}{12} \frac{\partial \widetilde{T}}{\partial x_{i}} \frac{\partial^{2} \widetilde{\psi}}{\partial x_{i} \partial x_{j}}, \\
q_{j}^{\psi} & =-\bar{\rho} \frac{\Delta_{c}}{2} \widetilde{k}^{1 / 2}\left(\delta_{i j}-e_{i}^{v} e_{j}^{v}\right) \frac{\partial \widetilde{\psi}}{\partial x_{i}}, \\
\phi^{T \psi} & =\bar{\rho} \frac{\Delta_{c}^{2}}{12} \frac{\partial \widetilde{T}}{\partial x_{i}} \frac{\partial \widetilde{\psi}}{\partial x_{i}},
\end{aligned}
$$

where $e_{i}^{v}$ are the direction cosines of the subgrid vortex axis. The implementation of the subgrid vortex model relies on the assumption of the alignment of $\boldsymbol{e}^{v}$ with extensional eigenvectors of the locally resolved-scale rate-of-strain tensor

$$
\widetilde{S}_{i j}=\frac{1}{2}\left(\frac{\partial \widetilde{u}_{i}}{\partial x_{j}}+\frac{\partial \widetilde{u}_{j}}{\partial x_{i}}\right)
$$

and/or with the resolved-scale vorticity (Kosovic et al. 2002). In (3.19a), (3.19b) and $(3.19 d)$, the subgrid kinetic energy $\widetilde{k}$, contained in the wavenumber range $k>\pi / \Delta_{c}$, is a function of the energy spectrum $E(k)$ of subgrid vortices of the stretched-spiral 
type proposed by Lundgren (1982):

$$
\widetilde{k}=\int_{\pi / \Delta_{c}}^{\infty} E(k) \mathrm{d} k=\int_{\pi / \Delta_{c}}^{\infty} K_{0} \varepsilon^{2 / 3} k^{-5 / 3} \exp \left(-\frac{2 k^{2} \widetilde{v}}{3|\widetilde{a}|}\right) \mathrm{d} k,
$$

where $K_{0}$ is the Kolmogorov pre-factor, $\varepsilon$ is the local cell-averaged dissipation, $\widetilde{a}=\widetilde{S}_{i j} e_{i}^{v} e_{j}^{v}$ is the axial strain along the subgrid vortex axis, and $\widetilde{v}=\bar{\mu} / \bar{\rho}$ is the filtered kinematic viscosity of the mixture. To complete the model, the group prefactor $K_{0} \varepsilon^{2 / 3}$ is calculated for each cell using a resolved-scale, second-order velocity structure function matching (Voelkl \& Pullin 2000; Pullin 2000). The second-order velocity structure function can be, for example, averaged over the surface of a sphere or a disk of radius $\Delta$. Typically, the separation scale $\Delta$ and the cutoff length scale $\Delta_{c}$ are taken to be the finest grid spacing $\Delta x$. In the present work, we use a $(y, z)$-circular structure function, in planes orthogonal to the base flow direction, to avoid the calculation of velocity component differences across existing shock waves. As explained in the Appendix, the subgrid term $(3.19 e)$ is in practice extremely small, even for the highest $A$ considered (mixture of air with $\mathrm{H}_{2}$ ), where the inclusion of this subgrid correction has shown no effect on the mixing-layer growth.

To time-evolve the conserved variables $\bar{\rho}, \bar{\rho} \widetilde{u}_{i}, \bar{E}$ and $\bar{\rho} \widetilde{\psi}$, the temperature field is first determined by locally solving the algebraic system of (3.16b) and (3.19e), starting from a guess given by $(3.16 b)$ when $\phi^{T \psi}=0$. From $\widetilde{T}$, compute $\bar{p}$ using $(3.16 a)$ and (3.19e), determine the temperature-dependent coefficients $\bar{\mu}, \bar{\kappa}$ and $\widetilde{D}$, and evaluate the terms $(3.14 b),(3.14 c)$ and $(3.19 b)$ appearing in the energy equation $(3.13 c)$.

In what follows, Favre-filtered quantities are identified with resolved-scale quantities computed in the LES, so overbars and tildes will be omitted.

\subsection{Adaptive mesh refinement framework}

The resolution requirements imposed by the physics of the flow vary greatly both spatially and temporally for these shock-driven turbulent simulations. In particular, around discontinuities in the flow (e.g. shock waves), any high-order shock-capturing scheme drops to first-order accuracy, and mesh refinement is an efficient way to reduce the numerical error around these sharp features. A parallel AMR framework written in object-oriented $\mathrm{C}++$ (AMROC), developed by Deiterding (Deiterding 2005; Deiterding et al. 2006), and based on the block-structured adaptive mesh refinement (SAMR) algorithm of Berger \& Oliger (1984) and Berger \& Colella (1989), proves to be advantageous for the LES of compressible flows such as RichtmyerMeshkov flows, where grid refinement is dynamically performed around the multiple moving shocks and the accelerated interface, while coarse resolution is sufficient elsewhere. SAMR methods provide large computational savings not only in the particular case of the planar RMI but also in other applications listed in Pantano et al. (2007).

\subsection{Hybrid numerical method}

The numerical method is formulated for uniform Cartesian grids and is effectively applied to each subgrid of the mesh hierarchy. The overall strategy is documented in Pantano et al. (2007) as an extension of the hybrid scheme by Hill \& Pullin (2004) to SAMR meshes with non-Cartesian embedded boundaries. A WENO scheme is used to capture discontinuities such as shock waves or to deal with impedance issues at fine/coarse mesh interfaces, but the scheme switches to a low-numerical dissipation, explicit, tuned centre-difference scheme (TCD) in the smooth or turbulent regions, which is optimal for the functioning of explicit LES such as the subgrid 
stretched-vortex method. As a tradeoff against accuracy, the TCD stencil is chosen to minimize truncation errors in LES in the sense of Ghosal $(1996,1999)$ and Hill \& Pullin (2004).

LES at very high Reynolds numbers using low-numerical dissipation, centred discretizations, raises the issue of numerical stability, since resolved and subgrid viscous dissipation sometimes provides negligible stabilization. For that reason, the momentum, energy and scalar convective terms are written in skew-symmetric form adapted to compressible flow (Blaisdell 1991). For the energy equation, here we use a formulation that conserves the variance in total internal energy (Honein \& Moin 2004).

The SAMR approach is based on flux discretizations. While WENO is naturally a flux-based formulation, the TCD scheme must be expressed in a flux form as well. Derivatives approximated by a difference operator can be written in a divergencelike flux difference at each cell centre. In particular, Pantano et al. (2007) describe the derivation of the flux corresponding to the derivative of products in a final skew-symmetric form.

\subsection{Time-marching method for SAMR}

The use of low-numerical dissipation centred schemes suitable for purely convective problems, as is the case for high-Reynolds-number flows, imposes particular temporal stability requirements. Explicit multi-stage schemes can be easily implemented within SAMR. Lower-order Runge-Kutta (RK) methods cannot stably convect neutral modes, so third- or fourth-order RK time-marching methods are considered. RK substages can also be unstable when using upwinding in WENO: all RK coefficients must be positive to avoid undesirable oscillations around shocks where WENO is used. As a result, the optimal third-order strong stability preserving (SSP) RK scheme of Gottlieb, Shu \& Tadmor (2001) is chosen. Details are provided in $\S 2$ of Pantano et al. (2007).

We have described an extension of the classical SAMR algorithm to meet the requirements of LES of compressible flows. The methodology has been validated against experiments on planar turbulent jets (Gutmark \& Wygnanski 1976; Pantano et al. 2007), planar RMI with reshock (Vetter \& Sturtevant 1995; Hill et al. 2006; Pantano et al. 2007) and converging shocks (Bond et al. 2009).

\subsection{Diagnostics}

We compute detailed space-time histories of plane averages (parallel to the incident shock) of base quantities, such as plane-averaged mass fraction, as well as more complex terms, such as resolved and subgrid turbulent dissipation. Using an angle bracket to denote a plane average in a $(y, z)$-plane at position $x$, and at time $t$, define for any quantity $X(x, y, z, t)$ the departures $X^{\prime}$ and $X^{\prime \prime}$ from plane averages and Favre-like plane averages as follows:

$$
X(x, y, z, t)=\langle X\rangle(x, t)+X^{\prime}(x, y, z, t)=\tilde{X}(x, t)+X^{\prime \prime}(x, y, z, t),
$$

where the tilde now represents the instantaneous Favre-like, plane-average:

$$
\widetilde{X}(x, t)=\frac{\langle\rho X\rangle}{\langle\rho\rangle} .
$$

The plane averages are performed over the length of the entire computational domain at a spacing consistent with the axial location of every grid cell on the finest level of refinement. The temporal sampling of statistics is performed at a significantly high 
frequency (about every 10 coarse time steps, i.e. a few hundreds of times over the duration of each simulation).

Mesh refinement and scheme switching each require flow-derived thresholds. For example, scaled density gradients are used in the present study to flag regions for refinement, and compression-based measures (Lombardini 2008) are employed to flag regions where WENO was needed to capture shocks. Each simulation was performed on the LANL Lobo cluster using 16 AMD Opteron $2.3 \mathrm{GHz}$, quad-core, quad-socket nodes (32 GB memory each) and consumed between 5000 and $15000 \mathrm{~h} \mathrm{CPU}$ time (depending on the case), statistics production included. Before the analysis of the results, we reported in table 1 the main computational parameters presented in this section. Table 4 lists, for each gas combination, basic information concerning the flow physics (e.g. local Atwood ratio, mixing-layer width and algebraic velocities) before and after the initial shock interaction, as well as around the primary reshock interaction and at late times.

\section{Flow description}

\subsection{Wave diagrams}

The wave diagrams for the heavy-light and light-heavy configurations are sketched in figure 2 for the case of an unperturbed interface and confirmed by figure 3, which superposes the position of the unperturbed interface (base flow) with the position of different planes related to $1 \%, 50 \%$ and $99 \%$ of plane-averaged heavy-fluid mass fractions $\langle\psi\rangle$, defining the commonly known spikes (structures of heavy fluid penetrating light fluid), centre and bubbles (structures of light fluid penetrating heavy fluid). Point-wise experimental data obtained from pressure transducer records and high-speed motion picture by Brouillette \& Sturtevant (1989, 1993, 1994) confirm the main features of the wave diagrams presented here. These plots show fundamental differences regarding the wave interactions following the first reshock event at $t=t_{\text {res }}$. Solutions of the Riemann problem show that a heavy-to-light shock-contact refraction produces a reflected expansion fan, while a shock wave is reflected in the case of a light-to-heavy refraction (see Lombardini \& Pullin 2009).

Therefore, the light-to-heavy reshock interaction following an initial heavy-to-light interaction produces a reflected shock travelling towards the endwall, reflecting off the wall and reshocking the interface a second time $\left(t=t_{\text {res } 2}\right)$. As seen in figure $2(a)$, successive reshock occurrences follow in a similar fashion, and with decreasing strength. Each reshock interaction impulsively supplies negative increments of axial velocity to the interface, as inferred from figure 4 for initially unperturbed or perturbed interfaces, bringing the interface to rest in a monotonic way. Each reshock is an impulsive event that (i) deposits vorticity by baroclinic torque across the mixing zone, and (ii) amplifies existing vorticity fluctuations.

The heavy-to-light reshock following an initial light-to-heavy interaction (figure $2 b$ ) generates a reflected expansion fan reflecting off the wall and re-interacting with the interface (from $t=t_{\exp }$ when the expansion front first impacts the interface). As a result of this interaction, a compression wave is reflected in the heavy-fluid region. After reflecting off the endwall, this compression wave refracts at the density interface, generating a reflected expansion wave in the heavy-fluid region as has already occurred during the reshock interaction. Expansion and compression waves of decreasing intensity alternately reverberate between the wall and the interface, causing a smooth inversion in the axial motion of the interface (cf. figure 4). These interactions continuously deposit energy into the mixing layer over the course of their 

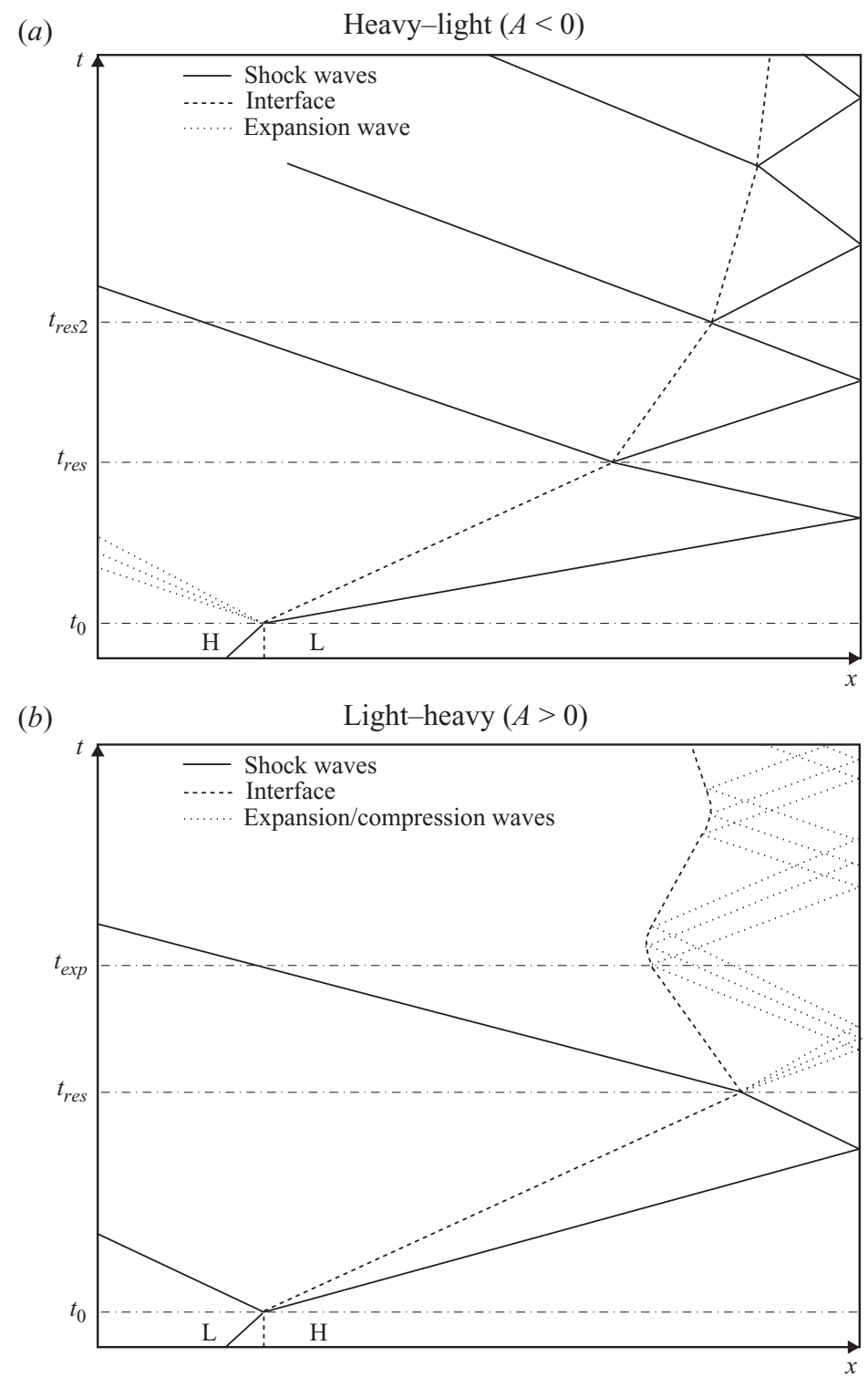

FIgURE 2. Approximate $(x, t)$ wave diagram for the interaction of a shock with $(a)$ a heavy-light $(A<0)$ and $(b)$ light-heavy $(A>0)$ density interface. The position of the unperturbed interface is drawn as well as the various wave reverberations following the first reshock. The initial shock interaction occurs at $t=t_{0}$; the first reshock is indicated by $t_{\text {res }}$, and the second wave interaction by $t_{\text {res } 2}$ in the heavy-light case (second reshock) and $t_{\text {exp }}$ in the light-heavy case (expansion).

passage from head to tail, as opposed to the impulsive deposition of energy due to the primary reshock or the successive reshocks in heavy-light configurations.

Note from figure 4 that, for a given $|A|$, the post-shock interface velocities for the corresponding light-to-heavy and heavy-to-light incident shock interactions equal each other, due to our choice of incident Mach numbers. 


\begin{tabular}{|c|c|c|c|c|c|c|c|}
\hline & Gas combination & Air- $-\mathrm{CO}_{2}$ & $\mathrm{CO}_{2}-$ air & Air- $-\mathrm{SF}_{6}$ & $\mathrm{SF}_{6}-$ air & $\mathrm{H}_{2}-$ air & Air $-\mathrm{H}_{2}$ \\
\hline \multirow{12}{*}{$\begin{array}{l}\text { Initial } \\
\text { conditions }\end{array}$} & Incident Mach number $M_{I}$ & 1.5 & 1.52 & 1.5 & 1.56 & 1.49 & 1.5 \\
\hline & Pre-shock Atwood ratio $A$ & 0.21 & -0.21 & 0.67 & -0.67 & 0.87 & -0.87 \\
\hline & Unshocked outer fluid density $\rho_{o}$ & 0.279 & 0.426 & 0.279 & 1.413 & 0.020 & 0.279 \\
\hline & Unshocked inner fluid density $\rho_{i}$ & 0.426 & 0.279 & 1.413 & 0.279 & 0.279 & 0.020 \\
\hline & Unshocked outer/inner fluid pressure $p_{o}$ & 23000 & 23000 & 23000 & 23000 & 23000 & 23000 \\
\hline & Unshocked outer/inner fluid temperature $T_{o}$ & 286 & 286 & 286 & 286 & 286 & 286 \\
\hline & Unshocked outer fluid sound speed $c_{o}$ & 340 & 264 & 340 & 133 & 1288 & 340 \\
\hline & Initial mixing-layer intrinsic thickness $L_{0}$ & 0.010 & 0.010 & 0.010 & 0.010 & 0.010 & 0.010 \\
\hline & Initial perturbation amplitude $\eta_{0}$ & 0.0025 & 0.0025 & 0.0025 & 0.0025 & 0.0025 & 0.0025 \\
\hline & Initial mixing-layer width $\delta_{0}$ & 0.020 & 0.020 & 0.020 & 0.020 & 0.020 & 0.020 \\
\hline & Dominant perturbation wavelength $\lambda_{0}$ & 0.027 & 0.027 & 0.027 & 0.027 & 0.027 & 0.027 \\
\hline & Initial membrane-centre-to-endwall distance & 0.62 & 0.62 & 0.62 & 0.62 & 0.62 & 0.62 \\
\hline \multirow{5}{*}{ Shock } & Post-shock interaction time $t_{0^{+}}$ & 0.00017 & 0.00019 & 0.00026 & 0.00045 & 0.000046 & 0.00016 \\
\hline & Post-shock Atwood ratio $A^{+}$ & 0.24 & -0.23 & 0.73 & -0.70 & 0.86 & -0.87 \\
\hline & Shocked interface velocity gain $\Delta u_{x}$ & 215 & 215 & 155 & 155 & 373 & 373 \\
\hline & Post-shock mixing-layer width $\delta_{0^{+}}$ & 0.011 & 0.012 & 0.011 & 0.012 & 0.010 & 0.015 \\
\hline & $\begin{array}{l}\text { Post-shock Richtmyer's growth rate } \\
\qquad 2 k \eta_{0^{+}}\left|A^{+}\right| \Delta u_{x} \text { if } \eta_{0^{+}} \simeq \eta_{0}\end{array}$ & 85 & 81 & 186 & 178 & 528 & 534 \\
\hline \multirow{8}{*}{$\begin{array}{l}\text { Primary } \\
\text { reshock }\end{array}$} & Reshock time $t_{r e s}$ & 0.0022 & 0.0020 & 0.0036 & 0.0025 & 0.0013 & 0.00078 \\
\hline & Post-reshock Atwood ratio $A_{\text {res }}{ }^{+}$ & 0.26 & -0.26 & 0.76 & -0.74 & 0.86 & -0.87 \\
\hline & Reshocked interface velocity gain $\Delta u_{x_{r e s}}$ & -238 & -195 & -213 & -100 & -588 & -156 \\
\hline & $\begin{array}{l}\text { Post-reshock } \rho \text {-weighted arithmetic mean } \\
\text { sound speed } \widehat{c}_{r e s}{ }^{+}\end{array}$ & 371 & 364 & 184 & 178 & 556 & 469 \\
\hline & Reshock Mach number $M_{r e s}$ & 1.49 & 1.39 & 1.72 & 1.28 & 1.66 & 1.18 \\
\hline & Pre-reshock mixing-layer width $\delta_{\text {res }}$ & 0.025 & 0.018 & 0.040 & 0.031 & 0.050 & 0.032 \\
\hline & Post-reshock mixing-layer width $\delta_{\text {res }}{ }^{+}$ & 0.014 & 0.010 & 0.020 & 0.025 & 0.030 & 0.031 \\
\hline & $\begin{array}{l}\text { Post-reshock growth rate estimated } \\
\text { from the computations }\end{array}$ & 8 & 9 & 50 & 30 & 170 & 85 \\
\hline \multirow{4}{*}{ Late flow } & Second wave interaction arrival time $t_{\text {exp }}$ or $t_{\text {res } 2}$ & 0.0031 & 0.0029 & 0.0046 & 0.0035 & 0.0019 & 0.0012 \\
\hline & Beginning of the TKE decay $t_{d e c}$ & 0.0052 & 0.0038 & 0.0059 & 0.0048 & 0.0025 & 0.0020 \\
\hline & $\begin{array}{l}\text { Approximate final membrane-centre-to-endwall } \\
\text { distance }\end{array}$ & 0.17 & 0.19 & 0.14 & 0.17 & 0.21 & 0.19 \\
\hline & Final Atwood ratio $A_{f}$ & 0.26 & -0.26 & 0.75 & -0.75 & 0.86 & -0.86 \\
\hline
\end{tabular}



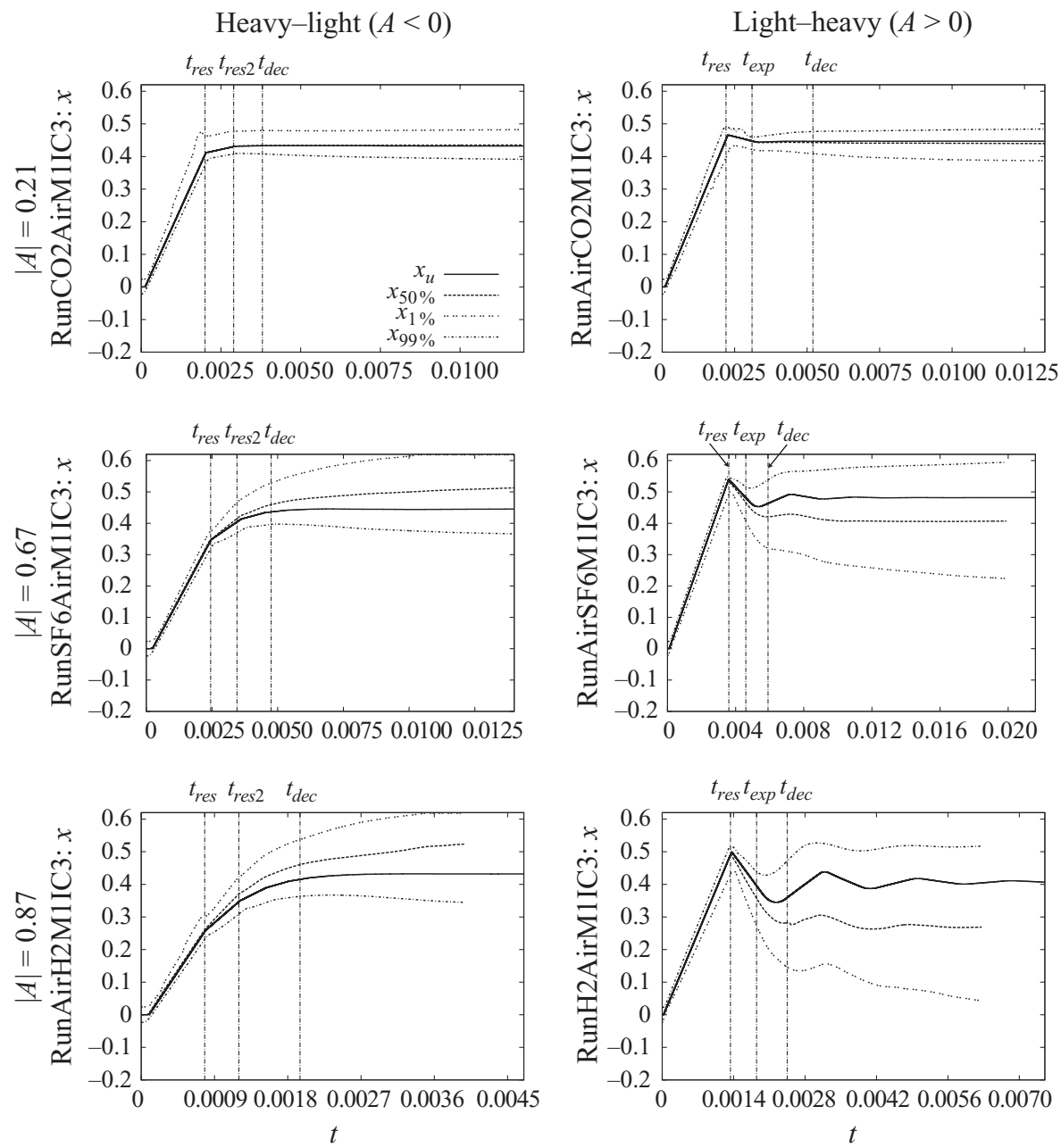

FIGURE 3. Axial position $x$ of the unperturbed interface $\left(x_{u}\right)$, mixing zone centre $\left(x_{50} \%\right)$, spike $\left(x_{1 \%}\right)$ and bubble $\left(x_{99} \%\right)$ structures (based on the plane-averaged heavy-fluid mass fraction $\langle\psi\rangle)$ versus $t$.

\subsection{Mixing-layer growth}

To measure mixing-zone growth, the mixture fraction $\langle\psi\rangle$ is plane-averaged and the width of the mixing region $\delta$ at time $t$ is defined according to

$$
\delta(t)=4 \int_{x_{\min }}^{x_{\max }}(1-\langle\psi\rangle)\langle\psi\rangle \mathrm{d} x .
$$

To interpret this definition, one can think of a smooth axial profile $\langle\psi\rangle$ of the mixture fraction from light fluid $(\langle\psi\rangle=0)$ to heavy fluid $(\langle\psi\rangle=1)$ in the form of a (symmetric) tanh function of intrinsic thickness $\delta$ and centred on the mixing-zone centre $x=x_{50} \%$. In this case, it is easy to show that (4.1) recovers this thickness, providing that the boundaries $x=x_{\min }$ and $x=x_{\max }$ are sufficiently far from $x_{50 \%}$. As a second measure of the mixing extent, the $1 \%$ and $99 \%$ edge positions, shown in figure 3, constitute a threshold-based definition of the width that accounts for the penetration of spikes commonly observed for large values of $|A|$ when the mixing 

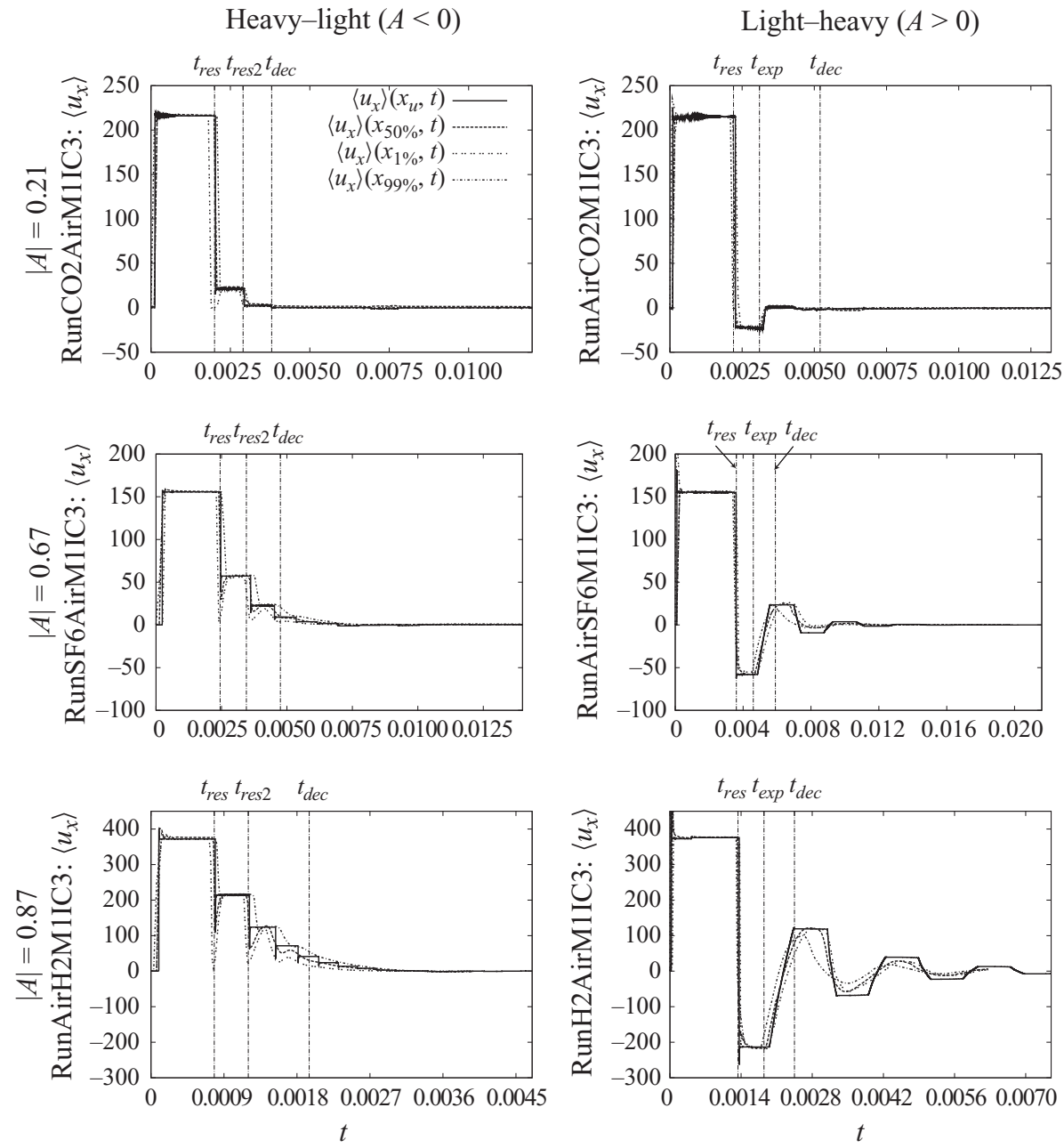

FIGURE 4. Plane-averaged axial velocity $\left\langle u_{x}\right\rangle$ of the unperturbed interface $\left(x_{u}\right)$, mixing zone centre $\left(x_{50} \%\right)$, spike $\left(x_{1 \%}\right)$ and bubble $\left(x_{99} \%\right)$ structures (based on $\left.\langle\psi\rangle\right)$ versus $t$.

zone grows asymmetrically. This definition complements the integral measure (4.1) that is advantageously independent of any arbitrary threshold.

Figure 5 shows the evolution of the width $\delta$ for each Atwood ratio, while figure 6 condenses, on a single plot, the mixing-layer width of every gas combination during the post-reshock phase of the flow. Monitoring the rate of change $\dot{\delta}$ is a useful diagnostic for identifying the various wave interactions following reshock. We first observe that at a given $|A|$, the widths and growth rates for heavy-light and light-heavy cases are of comparable amplitude. More precisely, for low values of $|A|$, the measured 'instantaneous' post-reshock growth rates are almost identical, but for larger $|A|$, the light-to-heavy post-reshock growth rate exceeds the heavy-to-light one, by a factor of two in the case $|A|=0.87$. We finally note that the strength of the secondary waves following the primary reshock increases as the density ratio increases.

There are fundamental differences between the heavy-light and light-heavy mixinglayer growth. In heavy-light configurations, the primary reshock greatly accelerates the growth initiated by the initial shock interaction, while subsequent wave interactions 

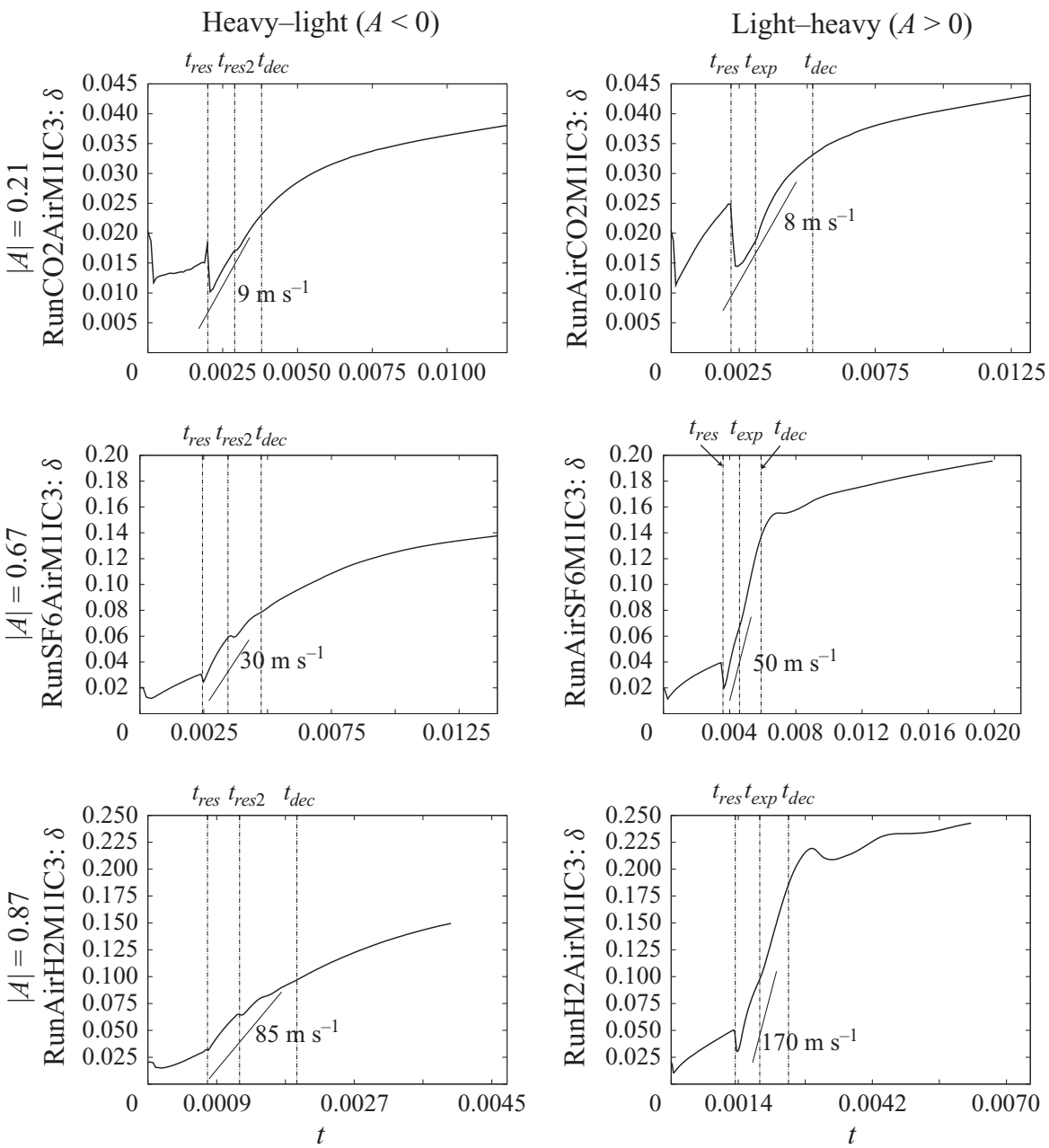

FIGURE 5. Mixing zone width $\delta$ versus $t$. The instantaneous post-reshock growth rate is estimated for each case.

have a minor effect on the growth amplitude. From figure 5, the post-reshock, finitetime evolution $\left(t>t_{\text {res }}\right)$ can be roughly characterized by an empirical $t^{\theta}$-relation, with $\theta(A)<1$. The light-heavy configurations can be described by two post-reshock phases: a post-reshock growth approximately linear in time, followed by a saturation phase occurring after 2-3 times the reshock time. The passage of the expansion and compression waves following the primary reshock has a stronger signature on the layer width (except perhaps in the case $A=0.21$ ) than the heavy-to-light reshocks. The segregation operated between the spikes and bubbles by these continuous reverberations responsible for such changes in the post-reshock mixing-layer growth is particularly visible in figure 3.

From figure 3, we roughly estimate that after about 4-5 times the reshock time, the successive wave interactions between the endwall and the layer become infinitesimally small in strength and the location of the turbulent mixing zone (TMZ) stabilizes at a certain distance from the endwall. However, the late-time self-evolving mixing zone 


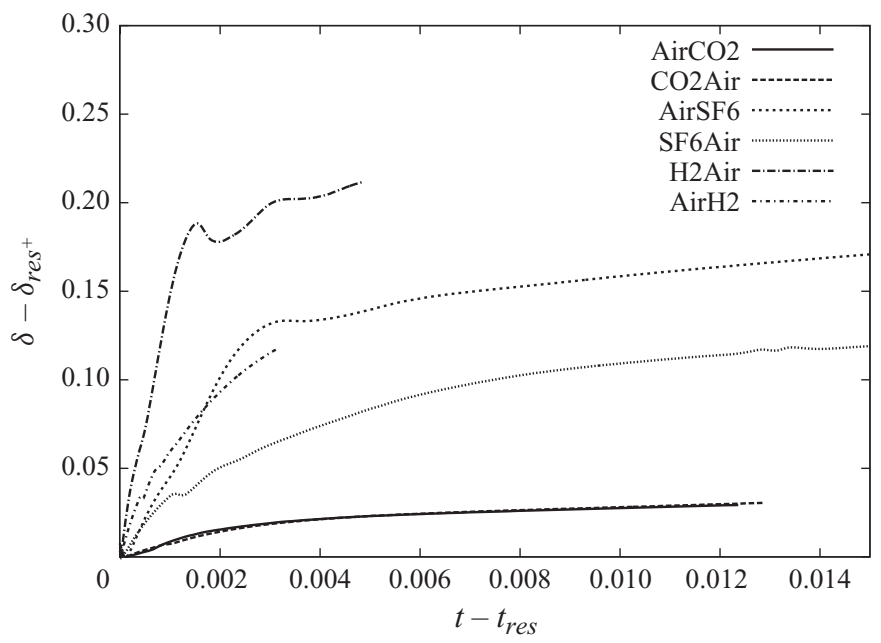

FIGURE 6. Post-reshock mixing zone width $\delta-\delta_{\text {res }}+$ versus $t-t_{\text {res }}$ for various gas combinations.

continues to grow (at a much slower rate than the post-reshock period) as a result of the on-going mixing activity, as confirmed by figures 5 and 6 .

\subsection{Turbulent kinetic energy and dissipation}

The successive wave interactions naturally stimulate the turbulent activity across the TMZ. This is illustrated in figure 7 which shows the evolution of the total volumeaveraged TKE and turbulent dissipation. To compute these quantities, Favre-like plane-averaged statistics of the turbulent activity such as resolved-scale TKE $\langle K\rangle$, subgrid-scale TKE $\langle k\rangle$ (per unit mass), resolved-scale dissipation $\left\langle\varepsilon_{\text {res }}\right\rangle$, and subgrid energy transfer $\left\langle\varepsilon_{\text {sgs }}\right\rangle$ are required:

$$
\begin{aligned}
\langle K\rangle & =\frac{1}{2} \operatorname{Var}_{\rho}\left(u_{i} u_{i}\right), \quad\langle k\rangle=\frac{\left\langle\tau_{i i}\right\rangle}{2\langle\rho\rangle}, \\
\left\langle\varepsilon_{r e s}\right\rangle & =\frac{\left\langle d_{i j}^{\prime} S_{i j}^{\prime}\right\rangle}{\langle\rho\rangle}, \quad\left\langle\varepsilon_{s g s}\right\rangle=-\frac{\left\langle\tau_{i j}^{\prime} S_{i j}^{\prime}\right\rangle}{\langle\rho\rangle},
\end{aligned}
$$

where we recall that $\tau_{i i}=2 \rho k$ from $(3.19 a)$. The volume averaging is achieved by adding and integrating the above resolved and subgrid plane averages in the direction of the shock tube axis. Note that $\langle\varepsilon\rangle-\left\langle\varepsilon_{\text {res }}\right\rangle \equiv\left\langle\varepsilon_{\text {sgs }}\right\rangle$, with $\langle\varepsilon\rangle$ being the total dissipation, represents the transfer of kinetic energy through the wave mode $\pi / \Delta_{c}$ and is provided by the stretched-vortex subgrid model using (3.19a). The total dissipation $\langle\varepsilon\rangle$ is simply related to the plane-average of $\sigma_{i j}^{\prime} S_{i j}^{\prime}$, where $\sigma_{i j}=d_{i j}-\tau_{i j}$ represents the total (resolved plus subgrid) stress tensor. We have the equalities

$$
\langle\varepsilon\rangle=\left\langle\varepsilon_{\text {res }}\right\rangle+\left\langle\varepsilon_{s g s}\right\rangle=\frac{1}{\langle\rho\rangle}\left(\left\langle d_{i j} S_{i j}\right\rangle-\left\langle d_{i j}\right\rangle\left\langle S_{i j}\right\rangle\right)-\frac{1}{\langle\rho\rangle}\left(\left\langle\tau_{i j} S_{i j}\right\rangle-\left\langle\tau_{i j}\right\rangle\left\langle S_{i j}\right\rangle\right) .
$$

Although only the total turbulent kinetic energy and dissipation are represented here, a breakdown of their respective resolved and subgrid contributions has shown the 

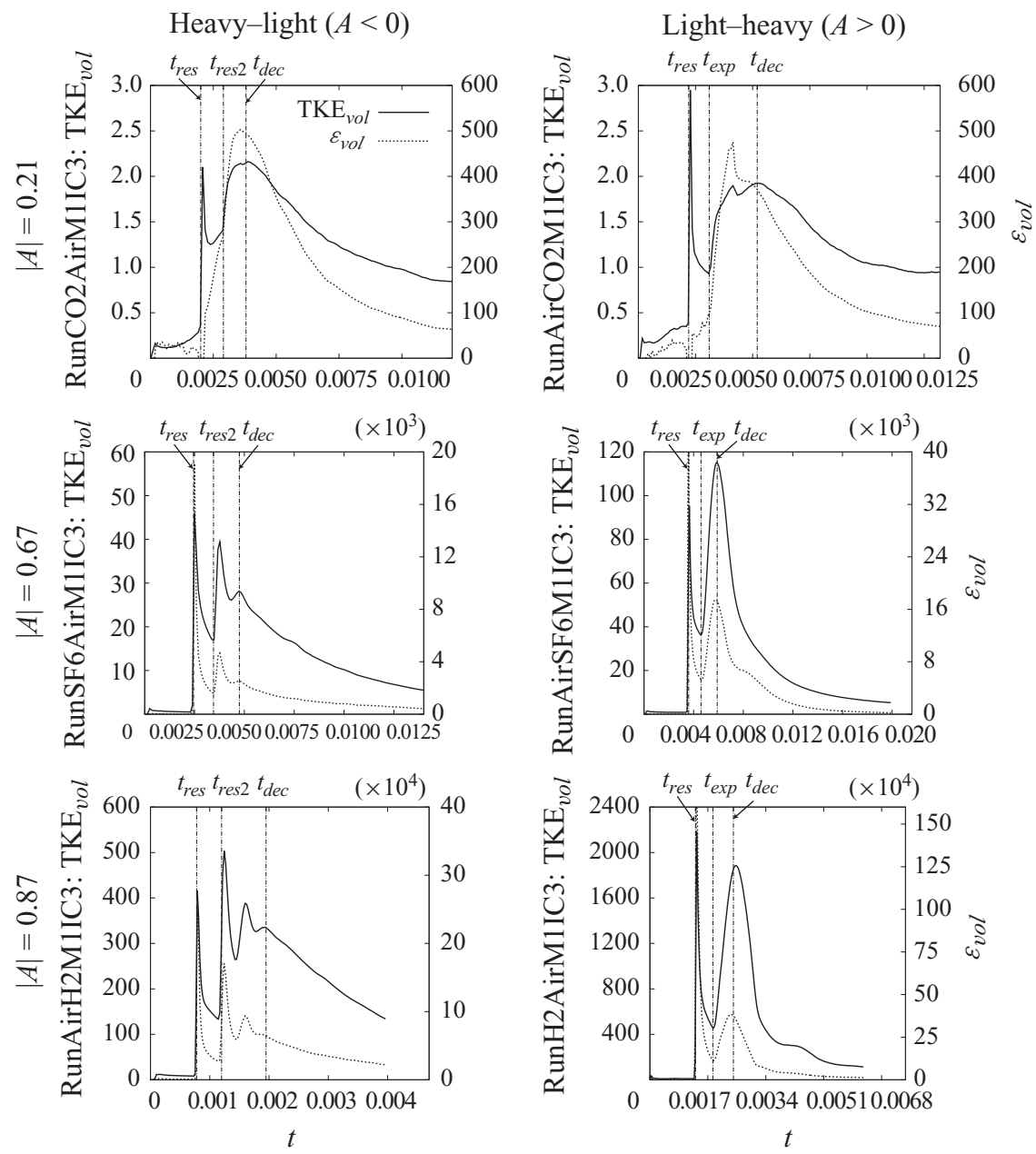

FIGURE 7. Volume-averaged resolved and subgrid TKE and turbulent dissipation versus $t$.

importance of the subgrid model in the post-reshock stages of the mixing (Hill et al. 2006).

Figure 7 summarizes the main historical events of the flow: first, the total TKE deposited by the initial shock, visible as a small bump near $t=0$, as well as that due to the first reshock around $t=t_{\text {res }}$. The reshock is more intense in the light-heavy cases than in the respective heavy-light cases, for each given $|A|$. A steep decay of TKE and a second interaction of comparable energy to that of the reshocking event follow the first reshock event: for $A<0$, the interaction is steep and corresponds to the second reshock; for $A>0$, the interaction is smoother in time and of higher intensity than its heavy-light counterpart. The increase in TKE following the reshock is accompanied by a similar increase in the turbulent dissipation. After about $2 t_{\text {res }}$, there is a very slow period of decay of TKE (defined by its starting time $t_{d e c}$ ) and turbulent dissipation, since none of the late reverberations coming from the wall posses sufficiently large pressure gradients across the mixing zone to sustain the turbulent activity. The late decline of TKE and turbulent dissipation appears delayed for $A<0$, while for the $A>0$ cases, it immediately follows the first expansion fan interaction and is characterized by a steeper rate of decay. These observations lead to 

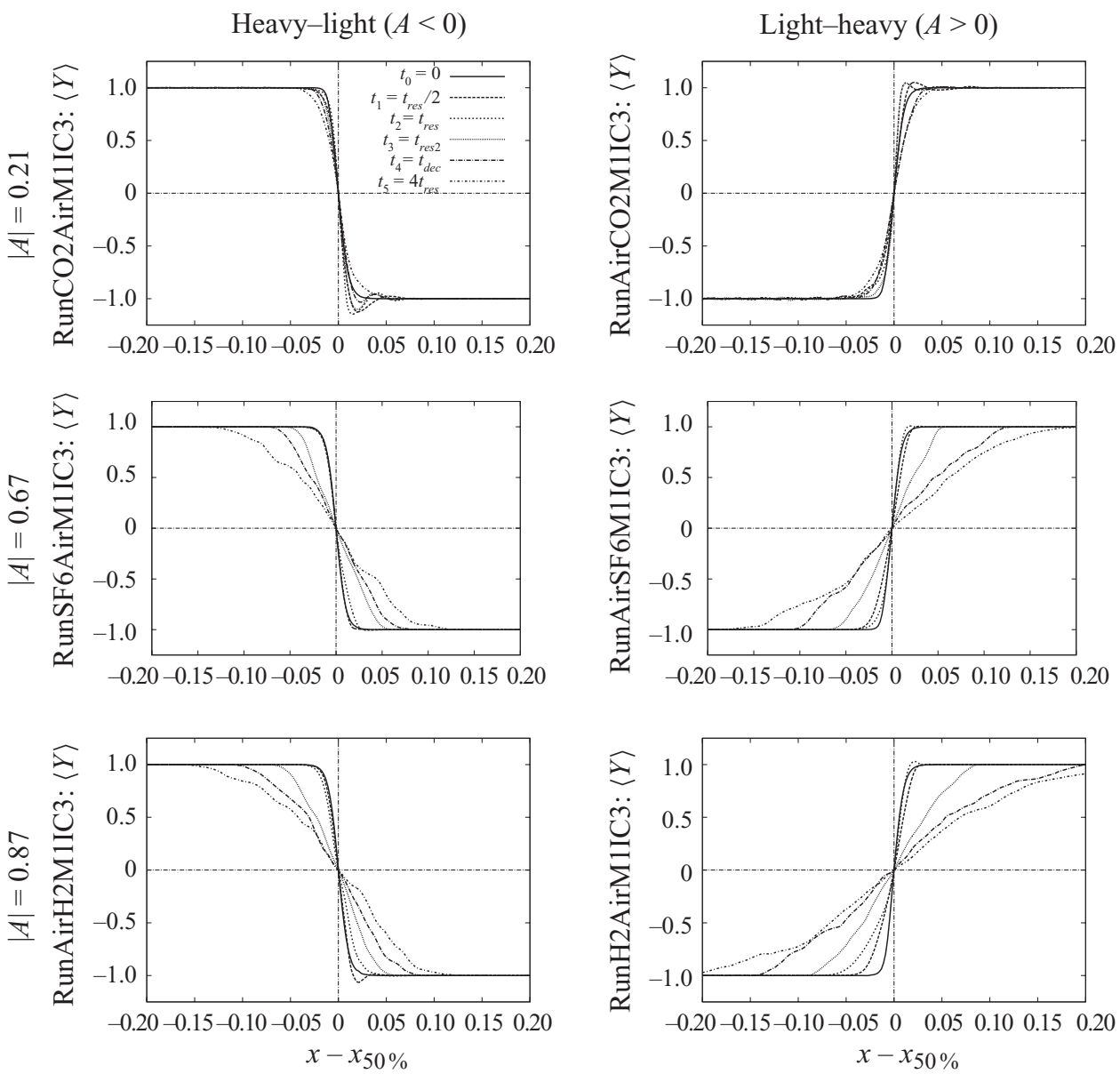

FiguRE 8. Plane-averaged scalar field $\langle Y\rangle \equiv 2\langle\psi\rangle-1$ versus $x-x_{50 \%}$, at six different times $t_{0}$, $t_{1}=t_{\text {res }} / 2, t_{2}=t_{\text {res }}, t_{3}=t_{\text {res } 2}$ or $t_{\text {exp }}, t_{4}=t_{\text {dec }}$ and $t_{5}=4 t_{\text {res }}$.

the first impression of a more sustained decaying turbulence activity within the mixing layer in the light-heavy configurations. Figure 7 also exhibits the effect of varying $|A|$. The amount of energy deposition during the successive wave interactions increases naturally as $|A|$ increases. For negative Atwood ratios with a large magnitude, the third (and even fourth) reshocks can be noticed.

\subsection{The inner structure of the mixing layer}

Figure 3 suggested an asymmetry in the mixing zone, as the spikes grow more than the bubbles measured from their unperturbed positions. As a consequence, the centre of the mixing zone drifts towards the light side of the flow. The asymmetry is naturally more pronounced for higher density ratios, as seen in figure 8, which shows planeaveraged mass fraction profiles at different stages of the flow. Dimonte \& Schneider (2000) summarized similar effects of the density ratio on the spike and bubble asymmetry for an impulsively accelerated interface (equivalent to a single shockinterface interaction): for low $|A|(|A| \lesssim 0.1)$, spikes and bubbles grow similarly, while for higher $|A|(|A| \gtrsim 0.8)$ spikes are long and narrow relative to the bubbles owing to vortex localization (Zabusky 1999; Dimonte \& Schneider 2000). 

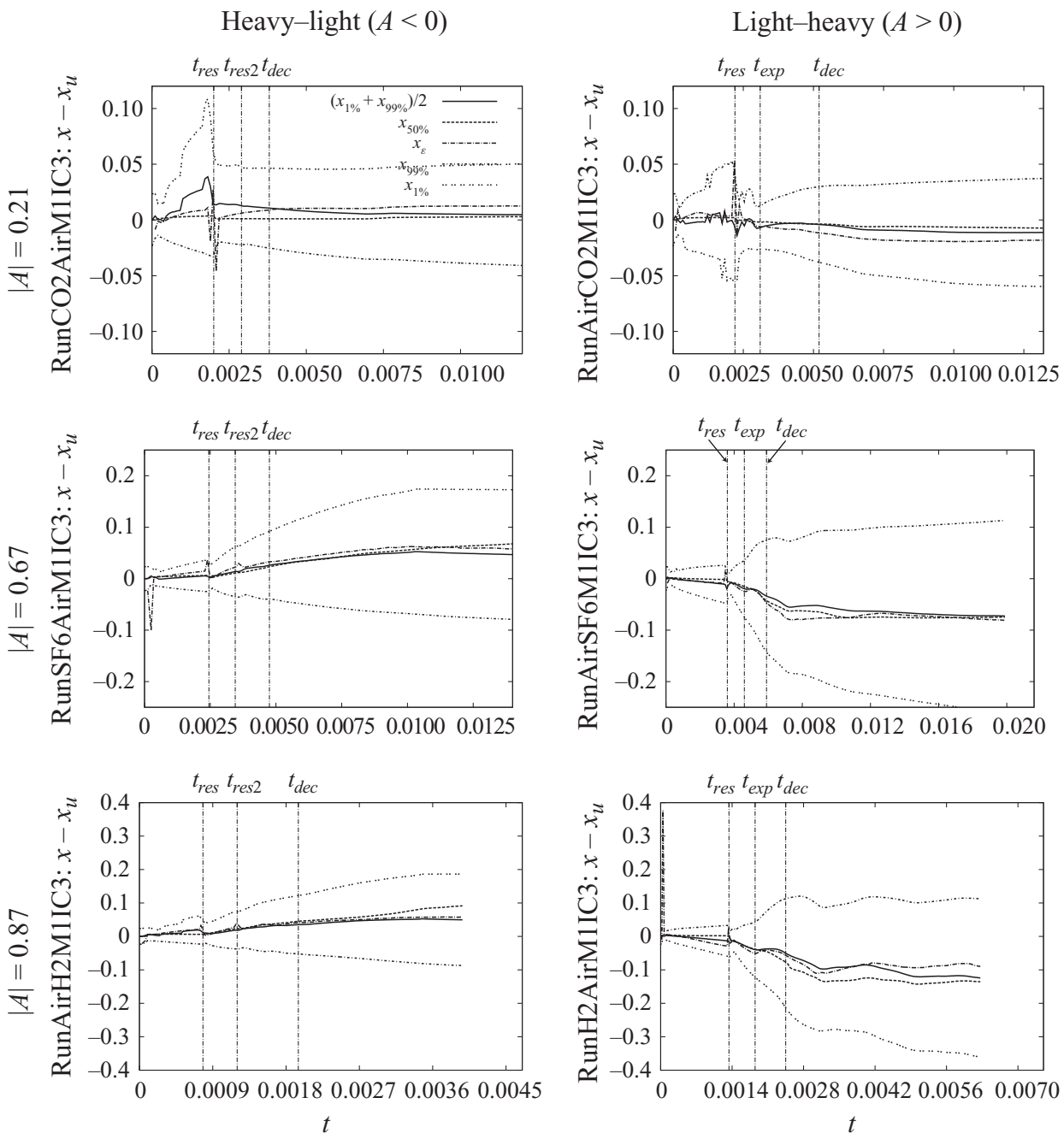

FIgURE 9. Axial positions $x-x_{u}$, in the frame of the unperturbed interface, of different quantities versus $t$ : geometric centre $\left(x_{1} \%+x_{99} \%\right) / 2 ;\langle\psi\rangle=50 \%$ position; centroid of the turbulent dissipation profiles; and envelopes $x_{1 \%}$ and $x_{99} \%$.

A more precise account of the lack of symmetry of the mixing layer is represented in figure 9, which displays the position of different planes across the mixing layer with respect to the unperturbed flow versus time. Figure 10 superposes the postreshock position of the mixing zone centre versus $\delta(t)$ for the six different values of the Atwood ratio. Both figures 9 and 10 show how the mixing zone centre deviates from its unperturbed position, always towards the light side. The deviation is more pronounced as $|A|$ increases. The geometric centre, defined as $\left(x_{1 \%}+x_{99} \%\right) / 2$, and the centroids of the turbulent dissipation profile

$$
x_{\varepsilon}(t)=\frac{\int_{x_{\min }}^{x_{\max }} x\langle\varepsilon\rangle \mathrm{d} x}{\int_{x_{\min }}^{x_{\max }}\langle\varepsilon\rangle \mathrm{d} x},
$$




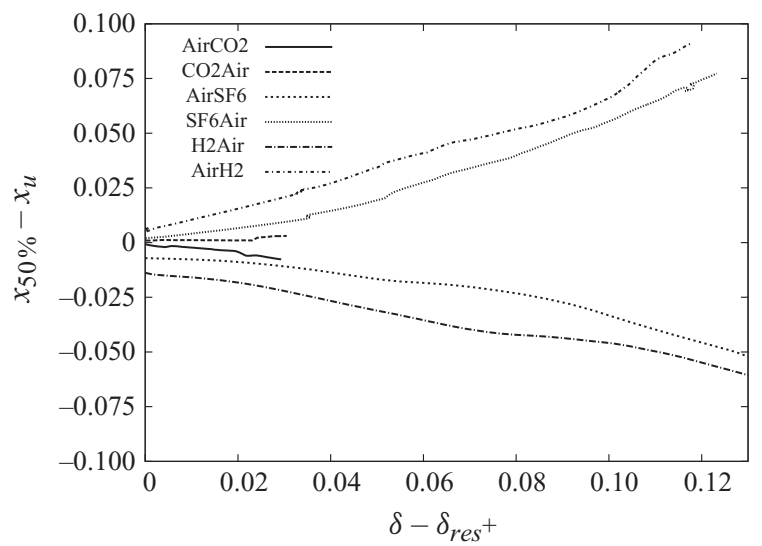

FIGURE 10. Post-reshock axial position $x_{50 \%}-x_{u}$ versus $\delta-\delta_{\text {res }}$ for various gas combinations.

are added to the definitions of spike, bubble and centre in figure 9 . We observe that centre $x_{50 \%}$, geometric centre, and $x_{\varepsilon}$ evolve distinctively. To better understand these observations, we plot in figures 11 and 12 the contour shades of the (normalized) TKE and turbulent dissipation profiles across the mixing zone as a function of time, for each Atwood ratio. For $A<0$, the peak of highest TKE and dissipation moves towards the light side of the fluid, as the centre $x_{50 \%}$ does. However, for $A>0$, the dominant regions of TKE and dissipation drift towards the heavy side compared with the centre $x_{50 \%}$. This effect is particularly visible at high $A>0$. This suggests that the expansion waves have a segregating role in the evolution of the mixing zone, where two mixing sub-regions would coexist, as Schilling et al. (2007) observed in their two-dimensional light-heavy simulations.

\section{On the reshock growth rate}

The goal of this section is to capture the Atwood ratio dependence of the instantaneous growth rate immediately after the reshock. After presenting the oftcited model of Mikaelian (1989), we derive another empirical law that operates for a wider range of Atwood ratios. A model based on the diffuse-interface approach of Saffman \& Meiron (1989) is then developed independently to clarify the influence of the Atwood ratio on the post-reshock growth.

\subsection{Empirical approach by Mikaelian (1989)}

The post-reshock growth rate measured from the simulations has been compared with the formula predicting a linear growth (in time) after the passage of a single shock through the turbulent mixing zone, with growth rate (Mikaelian 1989)

$$
\dot{\delta}_{\text {Mik. }}=0.28\left|A_{\text {res }^{+}}\right|\left(-\Delta u_{x_{\text {res }}}\right),
$$

where $A_{r^{+} s^{+}}$is the Atwood ratio immediately after reshock and $\Delta u_{x_{\text {res }}}<0$ is the axial velocity increment of the interface due to the reshock interaction. The model of Mikaelian (1989) consists of two steps. First, it incorporates RMI observations on the bubble amplitude evolution $\delta_{B}(t)$ in the RTI light-heavy experiments conducted by Read (1984), where $\delta_{B}=\alpha_{B} A g t^{2}, g$ being the acceleration of the two-fluid system. A quasi-constant coefficient $\alpha_{B} \approx 0.07$ was reported as $A \approx 0.23,0.50$ and 0.99 were tested. Second, the amplitude of the mixing zone $\delta=\delta_{B}+\delta_{S}$ is computed 

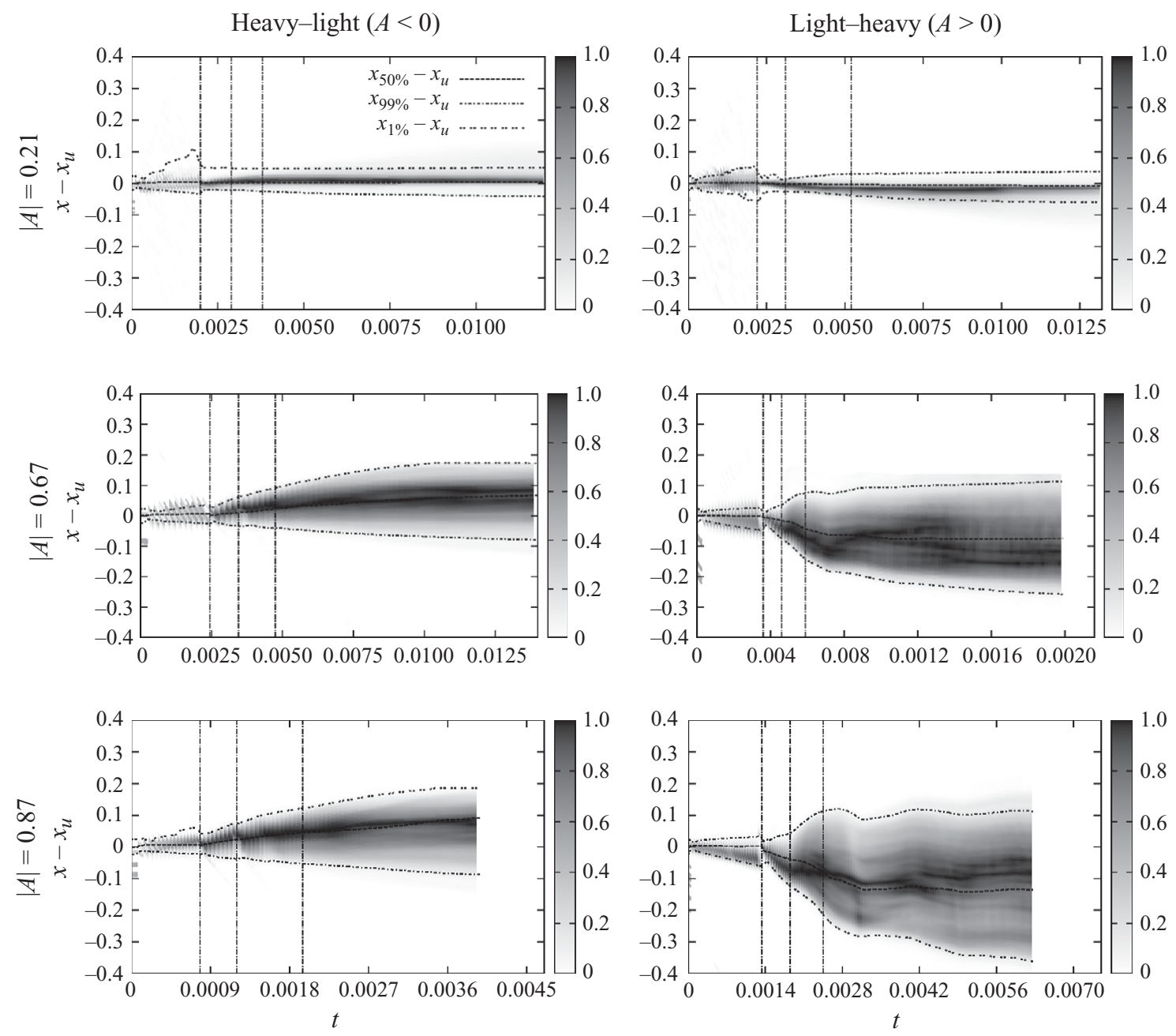

20

FIGURE 11. Contour shades of the normalized, plane-averaged, resolved and subgrid TKE in the $\left(x-x_{u}, t\right)$ domain. For each plot, the three dash-dotted vertical lines indicate the passage of the primary reshock, second wave interaction and the beginning of the TKE decay. 

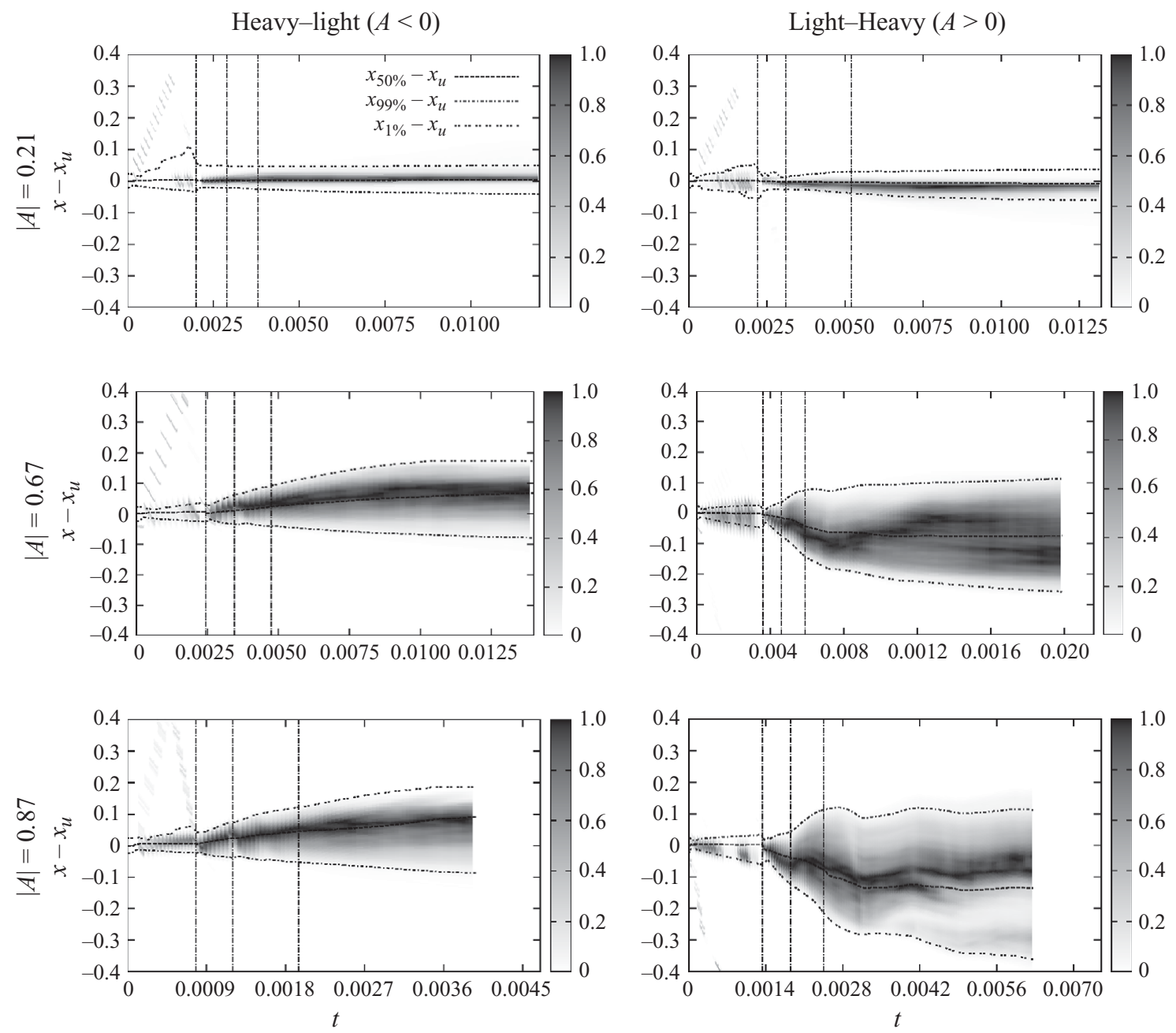

FIGURE 12. Contour shades of the normalized, plane-averaged, resolved and subgrid turbulent dissipation in the $\left(x-x_{u}, t\right)$ domain. For each plot, $\vec{\partial}$ the three dash-dotted vertical lines indicate the passage of the primary reshock, second wave interaction and the beginning of the TKE decay. 


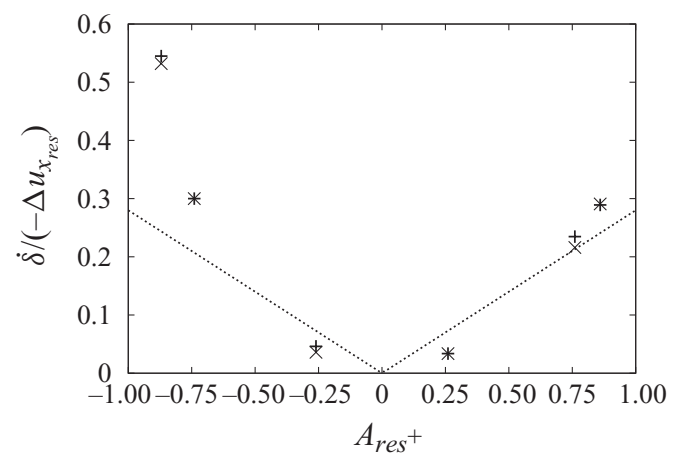

FIGURE 13. Normalized instantaneous post-reshock growth rate $\dot{\delta} /\left(-\Delta u_{\text {res }_{\text {res }}}\right)$ versus $A_{\text {res }}{ }^{+}$. The growth rate measured from the computations is designated by the ' + ' symbols, and the dashed line designates the prediction $0.28\left|A_{\text {res }^{+}}\right|$given by (5.1). The ' $\times$' symbols represent values of the growth rate given by the empirical law (5.3) evaluated for the gas combinations considered.

assuming symmetric evolution between the spike and bubble, $\delta_{B}=\delta_{S}$. Using both steps, differentiating twice in time gives $\ddot{\delta}=0.28 g \mathrm{~A}$ in the RTI and this is translated to the RMI by impulsive identification $g=\Delta u_{x} \delta(t)$ implying the result (5.1). An absolute value is used in (5.1) because the definition (4.1) proscribes negative values of the width (phase inversion in heavy-to-light interactions could instead be depicted by a spike-to-bubble definition of the width where no absolute value of the Atwood ratio is needed).

Figure 13 and table 5 show that notable discrepancies exist between the measured instantaneous post-reshock growth rate and the model (5.1) in most cases, although the latter works reasonably well for the two positive higher Atwood ratios. Several reshock experiments have suggested that the empirical coefficient in (5.1) should implicitly depend on $A$ (Brouillette \& Sturtevant 1993; Vetter \& Sturtevant 1995; Leinov et al. 2009, for air- $\mathrm{SF}_{6}$ as well as for other gas pairings). For example, Dimonte \& Schneider (2000) showed that, for a single impulsive acceleration, the bubble and spike amplitude each obeys a distinct power law $t^{\theta_{B, S}}$ with $\theta_{B} \approx 0.05$ and $\theta_{S}(A)$, while for constant accelerations $\delta_{B, S} \simeq \alpha_{B, S} A g t^{2}$ with $\alpha_{B} \approx 0.05$ and $\alpha_{S}(A)$. In particular, at high $|A|, \delta_{B}$ and $\delta_{S}$ drift from each other. Following the modelling approach of Mikaelian (1989), but using the results of Dimonte \& Schneider (2000) and Oron et al. (2001), suggests that the growth coefficient in (5.1) should be in the range of $0.28-0.40$.

\subsection{A new empirical law}

RMI growth rate models are often assumed to be of the form $\dot{\delta} / \Delta u_{x}=f\left(M_{I}, k \eta^{+}, A^{+}\right)$, where $f$ is a functional dependence to determine, but other dimensionless parameters could be included, e.g. the ratio of $\Delta u_{x}$ to a characteristic sound speed. In order to capture the strict influence of the Atwood ratio on the reshock growth rate $\left(M_{I}\right.$ and $k \eta^{+}$being fixed), we propose a simple empirical formulation where the instantaneous post-reshock growth rate is proportional to $\Delta u_{x_{\text {res }}}$, as in Richtmyer's impulsive model. This involves dimensionless parameters through the product of powers of $A_{\text {res }}{ }^{+}$and $\Delta u_{x_{\text {res }}} / \widehat{c}_{\text {res }^{+}}$, where $\widehat{c}_{\text {res }}{ }^{+}$is the post-reshock density-weighted average sound speed:

$$
\widehat{c}_{\text {res }}+=\frac{\rho_{o, r e s^{+}} c_{o, \text { res }^{+}}+\rho_{i, r e s^{+}} c_{i, r e s^{+}}}{\rho_{o, \text { res }^{+}}+\rho_{i, r e s^{+}}},
$$




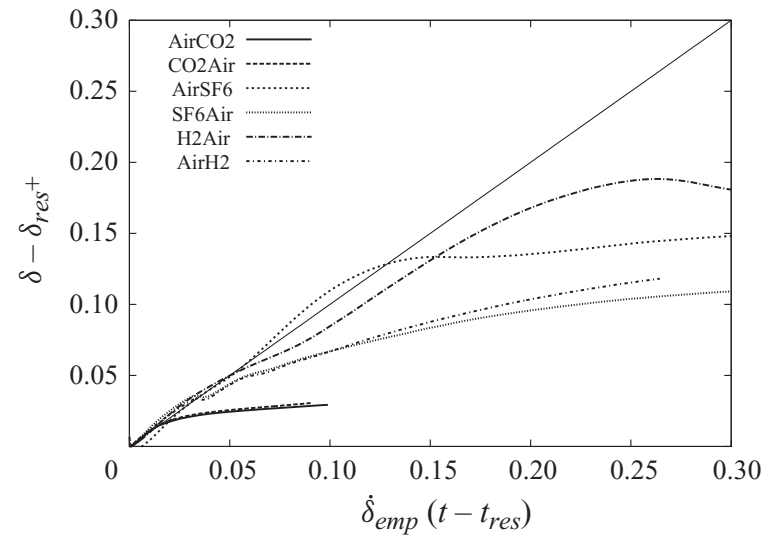

FIGURE 14. Post-reshock mixing zone width $\delta-\delta_{\text {res }}+$ versus $\dot{\delta}_{e m p}\left(t-t_{\text {res }}\right)$ for various gas combinations. The solid straight line represents the amplitude evolution following the empirical growth rate given by $(5.3)$.

defined as the ratio of the arithmetic mean of acoustic impedances and the mean of densities of the post-reshocked states of the fluid on each side of the interface. From the triplet $\left(\dot{\delta}, A_{r e s^{+}}, \Delta u_{x_{r e s}} / \widehat{c}_{r e s^{+}}\right)$measured from the plane-averaged scalar, density, velocity and temperature fields for each of the six gas combinations (the couple $\left(A_{\text {res }}+, \Delta u_{x_{\text {res }}} / \widehat{c}_{\text {res }}+\right)$ could also be determined by solving the equivalent one-dimensional shock-interface interaction), we find the least-squares fit

$$
\dot{\delta}_{\text {emp }} \simeq 0.40\left(\frac{-\Delta u_{x_{\text {res }}}}{\widehat{c}_{\text {res }}{ }^{+}}\right)^{-0.56} A_{\text {res }^{+}}^{1.96}\left(-\Delta u_{x_{\text {res }}}\right) .
$$

We recall that this model was obtained at a fixed incident Mach number and amplitude-to-wavelength ratio. The results for the post-reshock growth rate, displayed in figure 13 and reported in table 5 for the six cases studied, indicate a nonlinear dependence in the Atwood ratio $\sim A_{\text {res }}^{2}$, and the influence of an additional parameter, in the form of a Mach number defined by $-\Delta u_{x_{\text {res }}} / \widehat{c}_{r e s^{+}}$, which also has a built-in dependence on the light- and heavy-fluid densities. The factor $\left(-\Delta u_{x_{\text {res }}} / \widehat{c}_{\text {res }}\right)^{-0.56}$, whose values are reported in table 5 , is $\approx 1$ for positive Atwood ratios, but deviates significantly from 1 for negative Atwood ratios, in particular Atwood ratios of large magnitude.

As a separate test of the effect of the parameter $-\Delta u_{x_{\text {res }}} / \widehat{c}_{\text {res }}+$ on the post-reshock growth rate, the $\gamma$ values were fictitiously multiplied by six and the incident Mach number set to $M_{I}=1.89$ for the case air- $\mathrm{SF}_{6}$, which produced practically the same $\Delta u_{x_{\text {res }}} \approx 215 \mathrm{~m} \mathrm{~s}^{-1}$ and $A_{\text {res }}+\approx 0.68$, but increased the sound speed to $\widehat{c}_{\text {res }}{ }^{+} \approx 1231 \mathrm{~m} \mathrm{~s}^{-1}$. The empirical model (5.3), which predicts $\dot{\delta}_{e m p} \approx 95 \mathrm{~m} \mathrm{~s}^{-1}$, slightly overestimated the growth rate measured immediately after the reshock from the simulation, while the model (5.1) gives $\dot{\delta}_{M i k} \approx 41 \mathrm{~m} \mathrm{~s}^{-1}$.

Figure 14 represents the mixing-layer width evolution for $t>t_{\text {res }}$, as in figure 6 , but now using an appropriate time scaling based on (5.3).

\subsection{A diffuse-interface semi-analytical model}

The reshock problem is simplified by considering the two-dimensional interaction of a shock with an already grown density interface. This approach assumes that the prereshock velocity field does not play an important role in determining the post-reshock 
growth rate. A small perturbation, of characteristic amplitude $\eta$, is superposed on the diffuse density profile, of spatial extent $L$. An analytical expression for the growth rate is finally obtained after linearization with small parameter $\sim \eta / L$, and is completed by an empirical estimate of $\eta / L$ at the reshock.

\subsubsection{Diffuse-interface approach based on Saffman \& Meiron (1989)}

A diffuse density interface is impulsively accelerated at a speed $-\Delta u_{x} \boldsymbol{e}_{x}$ at $t=t_{\text {res }}$, with $\Delta u_{x}>0$. At $t=t_{\text {res }^{-}}$, the density is taken to be

$$
\rho_{0}=\bar{\rho}(x)+\rho^{\prime}(x, y)
$$

where the base density $\bar{\rho} \rightarrow \rho_{1,2}$ and the perturbation density $\rho^{\prime} \rightarrow 0$ as $x \rightarrow \pm \infty$. As a model of the initial density field, consider the following undisturbed density field and even-function single-mode perturbation density field:

$$
\bar{\rho}=\widetilde{\rho}[1+A \tanh (\xi)], \quad \rho^{\prime}=\epsilon \frac{\widetilde{\rho}}{2} \operatorname{sech}^{2}(\xi) \cos (k y),
$$

where $\widetilde{\rho}=\left(\rho_{1}+\rho_{2}\right) / 2$ is the average base density, $A=\left(\rho_{1}-\rho_{2}\right) /\left(\rho_{1}+\rho_{2}\right)$ is the Atwood ratio, and $\xi=x / L$ is the dimensionless axial coordinate, with $L$ being the characteristic width of the density profile $\bar{\rho}$. We also define the dimensionless parameter of linearization $\epsilon \equiv(2 \eta / L) A$, where $2 \eta$ is the peak-to-trough displacement of the constant density lines (density deformation profile $\zeta=\eta \cos (k y)$ ). The quantity $\eta$ is difficult to determine experimentally or computationally except in the limit of a discontinuous interface.

We enumerate some typical limit cases:

(a) $k \eta \ll 1$ : the classically small-amplitude, or linear, regime of the RMI;

(b) $\eta \ll L$ or $\epsilon \ll 1$ : small-amplitude perturbation compared with the interface width;

(c) $k L \ll 1$ : discontinuous interface;

(d) $k L \gg 1$ : diffuse interface.

It is expected that, around the reshock, the width of the already grown interface is essentially $\delta \simeq L+2 \eta$. As $\epsilon \ll 1, \delta \simeq L$. However, the growth rate after the impulse is inherently $\dot{\delta} \simeq 2 \dot{\eta}$. The values of $A, L$ and $\eta$, which parametrize the flow immediately following the impulse, will ultimately be related to their instantaneous post-reshock equivalents. We omit the 'res' subscript until required.

The initial base motion is a balance between the impulsive acceleration $-\Delta u_{x} \delta_{D}\left(t-t_{\text {res }}\right)$ and the pressure gradient (convective and viscous terms are negligible). For small $\epsilon$, Saffman \& Meiron (1989) showed that the axial velocity field, expressed in the frame of the moving interface (zero base velocity), is given by

$$
u_{x}^{\prime}=\frac{1}{\bar{\rho}}\left(\Delta u_{x} \rho^{\prime}-\frac{\partial p^{\prime}}{\partial x}\right)
$$

where the pressure perturbation $p^{\prime}$ is governed by the differential equation in $\xi$ :

$$
\frac{\partial}{\partial \xi}\left(\frac{1}{\bar{\rho}} \frac{\partial p^{\prime}}{\partial \xi}\right)-\frac{k^{2} L^{2}}{\bar{\rho}} p^{\prime}=L \Delta u_{x} \frac{\partial}{\partial \xi}\left(\frac{\rho^{\prime}}{\bar{\rho}}\right)
$$

with $p^{\prime} \rightarrow 0$ as $\xi \rightarrow \pm \infty$.

To define, for general base density profiles, the growth rate of an interface perturbation of the form $\zeta=\eta \cos (k y)$, we first observe that the perturbation amplitude 
is given by

$$
2\left(\rho_{2}-\rho_{1}\right) \eta=\frac{k}{2} \int_{-\pi / 2 k}^{\pi / 2 k} \int_{0^{-}}^{\infty} \rho^{\prime} \mathrm{d} x \mathrm{~d} y-\frac{k}{2} \int_{-3 \pi / 2 k}^{-\pi / 2 k} \int_{-\infty}^{0^{+}} \rho^{\prime} \mathrm{d} x \mathrm{~d} y,
$$

where $x=0$ corresponds to the interface centre position at rest. In (5.8), the region $\zeta<0$ (resp. $>0)$ when $k \in[-3 \pi / k,-\pi /(2 k)]$ (resp. $[-\pi /(2 k), \pi /(2 k)])$, modulo $2 \pi / k$, corresponds to the region of fluid 1 (resp.2) penetrating into fluid 2 (resp.fluid 1). Differentiating (5.8) with respect to $t$ and using the linearized continuity equation for incompressible flows in the frame of the accelerated interface, $\partial \rho^{\prime} / \partial t=-u_{x}^{\prime} \mathrm{d} \bar{\rho} / \mathrm{d} x$, the growth rate is obtained as a function of the axial velocity perturbation and base density:

$$
\dot{\delta}=\frac{k}{2\left(\rho_{1}-\rho_{2}\right)}\left[\int_{-\pi / 2 k}^{\pi / 2 k} \int_{0^{-}}^{\infty} \frac{\mathrm{d} \bar{\rho}}{\mathrm{d} x} u_{x}^{\prime} \mathrm{d} x \mathrm{~d} y-\int_{-3 \pi / 2 k}^{-\pi / 2 k} \int_{-\infty}^{0^{+}} \frac{\mathrm{d} \bar{\rho}}{\mathrm{d} x} u_{x}^{\prime} \mathrm{d} x \mathrm{~d} y\right] .
$$

\subsubsection{Discontinuous-interface limit $k L \ll 1$}

In this limit, the equivalent base and perturbation density profiles reduce to

$$
\bar{\rho}=\widetilde{\rho}[1-A+2 A H(x)], \quad \rho^{\prime}=2 \widetilde{\rho} A[H(x-\zeta)-H(x)],
$$

where $H$ is the Heaviside function. Employing the method of matched asymptotic expansions for a solution of (5.7) of the form $p^{\prime}=P(\xi) \cos (k y)$ as $k L \ll 1$, and then using (5.6), Saffman \& Meiron (1989) found the axial velocity perturbation field $u_{x}^{\prime}=k \eta \Delta u_{x} \mathrm{e}^{k|x|} \cos (k y)$. The growth rate (5.9) for a discontinuous interface then reduces to $\dot{\delta}_{d i s c .}=2 k \eta A \Delta u_{x}$, and the asymptotic growth rate for single-mode smallamplitude perturbations at discontinuous interfaces modelled by Richtmyer (1960) is naturally recovered.

\subsubsection{Diffuse-interface limit $k L \gg 1$}

In this limit, the solution $p^{\prime}$ to (5.7) is well approximated by retaining the second term on the left-hand side of this equation, and depends on $\bar{\rho}$ and $\rho^{\prime}$ modelled by (5.5). Then, from (5.6),

$$
\begin{gathered}
u_{x}^{\prime}=\frac{\eta}{L} \Delta u_{x} A F(\xi) \cos (k y), \\
F(\xi)=\frac{1}{1+A \tanh (\xi)}\left(\operatorname{sech}^{2}(\xi)+\frac{1}{k^{2} L^{2}} \frac{\mathrm{d}}{\mathrm{d} \xi}\right. \\
\left.\times\left\{[1+A \tanh (\xi)] \frac{\mathrm{d}}{\mathrm{d} \xi}\left[\frac{\operatorname{sech}^{2}(\xi)}{1+A \tanh (\xi)}\right]\right\}\right) .
\end{gathered}
$$

Note that $u_{x}^{\prime}$ is not an even function of $\xi$, contrary to the discontinuous-interface limit. This asymmetry in the velocity eigenfunction for diffuse interfaces, already observed by Mikaelian (1991), is responsible for a more complex dependence of the growth rate on the Atwood ratio than in the case of sharp interfaces. Using (5.5) and (5.11), and after some algebraic manipulations, the growth rate (5.9) of small-amplitude perturbations at a diffuse interface simplifies to

$$
\dot{\delta}_{\text {diff }} \simeq \frac{\eta}{\delta}|A| g(A) \Delta u_{x}, \quad g(A)=\frac{1}{A^{2}}\left[1-\frac{1-A^{2}}{2 A} \log \left(\frac{1+A}{1-A}\right)\right],
$$

where the absolute value accommodates the definition of the width (4.1), $g(A)$ being an even function of $A$. 
This growth rate tends to zero if $\epsilon \ll 1$ (small-perturbation limit), $A \ll 1$ or for infinitely weak shocks (i.e. $\Delta u_{x}=0$ ). Since $g(A)<1$ for $|A|<1$, the growth rate of a diffuse interface is reduced by a factor $\Phi \equiv 2 k \delta / g(A)>1$ compared with Richtmyer's growth rate, where $g(A)$ is an increasing function of $|A|$. Note that the value of $\Phi$ increases monotonically with increasing interface thickness. Our model follows the observations of Brouillette \& Sturtevant (1994), which in turn are confirmed by the experiments of Collins \& Jacobs (2002) on air- $\mathrm{SF}_{6}$ diffuse interfaces single-shocked at incident Mach numbers 1.11 and 1.21. Brouillette \& Sturtevant (1994) noticed a reduction factor for shock-accelerated diffuse interfaces $\Psi \simeq k \delta C(A) /(2 \pi)>1$, where $C(A)$ is a decreasing function of $A$, for $A>0$. Values of $C(A)$ need to be computed numerically by solving a Sturm-Liouville boundary-value problem for the eigenvalue $\Psi$ given an initial density distribution (Duff, Harlow \& Hirt 1962; Brouillette \& Sturtevant 1994). The same eigenvalue problem is the starting point of an analysis by Mikaelian (1991) in which the growth rate, associated with the fastest growing eigenmode, is found after approximating this eigenmode by Richtmyer's velocity eigenfunction given in $\S 5.3 .2$. In the diffuse limit, $k L \gg 1$, his growth rate scales like $(\eta / \delta)|A| \Delta u_{x}$ for arbitrary values of $A$, which coincides with the small- $A$ limit of (5.12).

\subsubsection{Closure of the diffuse-interface model}

The previous impulsive approach recovers Richtmyer's growth rate in the discontinuous-interface limit $(k L \ll 1)$, which is known to work well for weak shocks impacting discontinuous interfaces. In the case of a diffuse interface $(k L \gg 1)$ as a description of the pre-reshocked layer, a simple law has been obtained at dominant order in $1 /(k L)$, given by (5.12). To complete this model, the $A$-dependence of $\eta / \delta$ is required around the reshock time, where $\eta$ is in fact the amplitude to which the perturbation has grown from its initial value $\eta_{0}$ until the reshock event. We directly estimated $\eta / \delta$ by measuring from the simulation data the root mean square (r.m.s.) plane-averaged density just before the reshock, and comparing with the r.m.s. given by the modelled density perturbation profile $(5.5 b)$ as

$$
\sqrt{\left\langle\rho^{\prime 2}\right\rangle}=\sqrt{\epsilon^{2} \frac{\widetilde{\rho}^{2}}{4} \operatorname{sech}^{4}(\xi)\left[\frac{1}{L_{y}} \int_{-L_{y} / 2}^{L_{y} / 2} \cos ^{2}(k y) \mathrm{d} y\right]}=\frac{1}{2 \sqrt{2}} \frac{\eta}{\delta}\left|\rho_{1}-\rho_{2}\right| \operatorname{sech}^{2}(\xi),
$$

where we used $L \simeq \delta$. In the above integral, the channel width $L_{y}$ is an integer number of the perturbation wavelength $2 \pi / k$. As inferred from figure $15, \eta / \delta$ is fairly well approximated by $C^{\prime}|A|^{\beta}$, with $\beta \approx 1$ and $C^{\prime}$ in the range of $0.58-0.62$. The expression (5.12) thus becomes

$$
\dot{\delta}_{\text {diff }} \simeq C^{\prime} A^{2} g(A) \Delta u_{x}, \quad g(A)=\frac{1}{A^{2}}\left[1-\frac{1-A^{2}}{2 A} \log \left(\frac{1+A}{1-A}\right)\right] .
$$

For small Atwood ratios, $\dot{\delta}_{\text {diff }} \sim A^{2} \Delta u_{x}$, reminiscent of the empirical $A^{2}$-dependence observed in $\S 5.1$. In this model, the dependence on the sign of $A$ (at fixed magnitude $|A|)$ is implicitly included in $\Delta u_{x}$, since $A^{2} g(A)$ is an even function of $A$.

In (5.14), $A$ is identified with the post-reshock Atwood ratio $A_{\text {res }}$ (i.e. $\rho_{1} \leftrightarrow \rho_{i}$ and $\rho_{2} \leftrightarrow \rho_{o}$ ) and $\Delta u_{x}$ with the velocity gain due to reshock, $-\Delta u_{x_{\text {res }}}$. The growth rate $\dot{\delta}_{\text {diff }}$ 


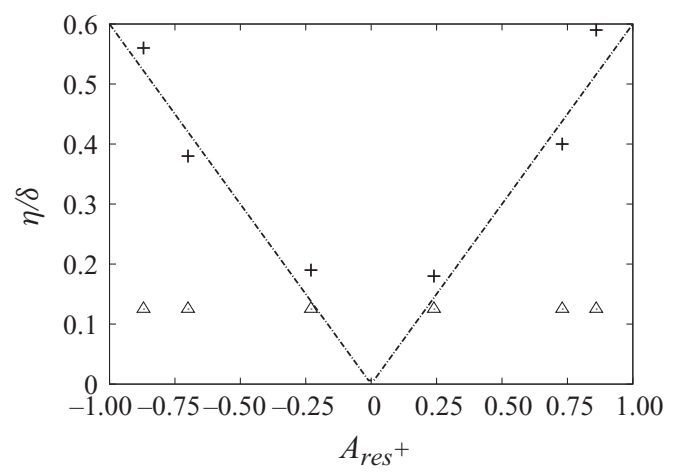

FIGURE 15. $\eta / \delta$ versus $A_{\text {res }}$. The symbols correspond to the measurement from the simulation data initially $(\triangle)$ and pre-reshock $(+)$, while the dash-dotted line indicates the appropriate fit $\sim\left|A_{\text {res }}\right|$ for the pre-reshock data.

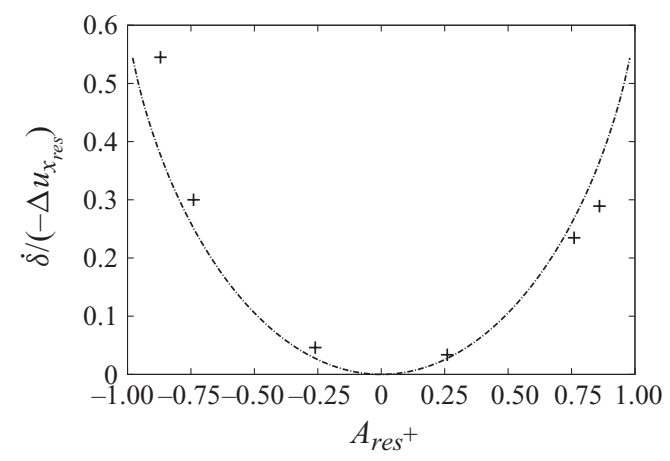

FIGURE 16. Normalized instantaneous post-reshock growth rate $\dot{\delta} /\left(-\Delta u_{x_{\text {res }}}\right)$ versus $A_{\text {res }}+$, measured from the computations ( + symbols) and given by the diffuse-interface model (dash-dotted line) given by (5.14).

is displayed in figure 16 and evaluated in table 5 for the six cases studied, showing reasonable agreement with the measured post-reshock growth rates.

The differences between measured and modelled reshock perturbation amplitudes can be partly explained by the uncertainty in sampling the density field at the correct reshock time. The evaluation of the dimensionless numbers $k \delta_{\text {res }^{+}}$and $\eta_{\text {res }^{+}} / \delta_{\text {res }^{+}}$, computed from the simulation and reported in table 5, allowed us to estimate the validity of the model's assumptions $k L \gg 1$ (diffuse interface) and $\eta / L \ll 1$ (small perturbations). The diffuse-interface assumption being actually $k^{2} L^{2} \gg 1$, considering (5.7), the first assumption is therefore valid for moderate and large values of $|A|$, for which the interface has grown sufficiently until the reshock. The second assumption would hold for low $|A|$ only. Deviations from these assumptions could partly explain the discrepancies observed between the diffuse-interface model and the measured growth rates. Figure 16 also indicates that large departures from the model (5.14) to the data occur for negative Atwood ratios, especially of large magnitudes. Consistent with the observations made at the end of $\S 5.2$, a model better than (5.14) would consist of replacing the constant $C^{\prime}$ by a function $C^{\prime}\left(\Delta u_{x_{r e s}} / \widehat{c}_{r e s}+\right)$, but this requires 
understanding corrections due to compressibility, which are not in the present diffuseinterface model (5.14).

\section{Conclusion}

We performed numerical computations of the RMI with reshock in the canonical geometry of a square cross-section, straight shock tube, with both cross-directions periodic. A parametric study was performed by varying the initial Atwood ratio $A$ both in sign and magnitude, employing a perturbation of the initial density interface with a given dominant wavelength. At given $|A|$, the matching of the initial shockcontact interaction strength, using Richtmyer's impulsive model, allows comparable scaling between each opposite-signed Atwood ratio.

The nature of the flow, involving a moving mixing region and travelling waves, and the long aspect ratio of the geometry justified the use of adaptive mesh refinement. The highly turbulent regime following the various compressions and/or expansions of the mixing region required the use of LES, the finest resolution being well within the inertial subrange when this exists (Hill et al. 2006).

Given our geometry of a closed shock tube, the sign of the Atwood ratio plays an important role in determining the mechanism by which additional TKE is deposited after reshock. In particular, we find that, in the light-heavy cases, there is more turbulent activity following the first expansion-wave interaction than occurs after the secondary reshocks in the heavy-light configuration. All cases lead to a late-time decay of TKE and turbulent dissipation. The mixing layer grows asymmetrically, and this effect is intensified as the Atwood ratio increases in magnitude. The contour shades of the TKE and turbulent dissipation highlight the bimodal nature of the mixing zone in the light-heavy configurations.

The post-reshock growth rate appears linear in time for a narrow period of time only. A first empirical law suggested a non-trivial dependence on the Atwood ratio and motivated the development of a second model for the reshock growth rate. Based on the impulsive growth of a slightly perturbed diffuse interface and an empirical closure, this model seems to capture the influence of the Atwood ratio on the reshock growth rate, in the way that the first empirical model does. An explicit $A^{2}$-dependence emerges in the range of small and moderate Atwood ratios. Since here we neglect compressibility in this model and do not consider the presence of a pre-reshock vorticity, our results suggest that, for weak or moderate incident shock strengths, both compressible and vorticity-amplification effects are of secondary importance for the post-reshock growth, but instead, simple baroclinic deposition at a diffuse interface is the dominant effect. This needs to be confirmed numerically by considering for example a set of different pre-reshock vorticity distributions. For strong incident shocks, however, the diffuse-interface description alone might prove incomplete.

Future work will consist of investigating in more detail the interaction of the first expansion wave and the post-reshock mixing layer, as occurs in light-heavy configurations. More gas combinations (e.g. $|A|=0.5$ and 0.95) and incident Mach numbers will also be tested and compared against the empirical and diffuse-interface models. The second part of the work presented here will describe the nature of the late-time turbulent mixing, raising issues such as the transition in shock-driven flows and the late-time decay of TKE.

This work has been supported in part by the Department of Energy under subcontract no. DE-AC52-06NA25396. 
Appendix. Subgrid model and scaling estimates for the correlations $\widetilde{T \psi}-\widetilde{T} \widetilde{\psi}$ and $\widetilde{T \partial_{j} \psi}-\widetilde{T} \partial_{j} \widetilde{\psi}$

To model the local correlation $\widetilde{T \psi}-\widetilde{T} \widetilde{\psi}$ in a two-fluid mixture, we propose, following Pullin (2000), an approximate solution for the winding of a scalar field by an elemental subgrid stretched-spiral vortex. This idea is applied not only to the mass fraction $\psi$ but also to the temperature field $T$, as was done by Kosovic et al. (2002) to evaluate $q_{j}^{T}$. Each field, e.g. $\psi$, is assumed to follow a simple convection equation, the effect of scalar diffusion compared to convection within a vortex being neglected. The solution within a subgrid cubic element of a characteristic side $\Delta_{c}$ can be written in the frame of the subgrid element (of origin the cell centre and fixed coordinates $x_{1}$, $x_{2}$ and $x_{3}$ ) using a Taylor series:

$$
\psi=\psi_{0}+\frac{\partial \widetilde{\psi}}{\partial x_{j}} x_{j}+\psi^{\prime \prime}
$$

where $\psi_{0}$ corresponds to $\psi$ evaluated at the cell centre. The fluctuation $\psi^{\prime \prime}$ is defined such that $\widetilde{\psi^{\prime \prime}}=0$, where the resolved-scale quantity $\widetilde{Q}$ is identified with the volume average of $Q$ within the subgrid element:

$$
\widetilde{Q}=\frac{1}{\Delta_{c}^{3}} \int_{-\Delta_{c} / 2}^{\Delta_{c} / 2} \int_{-\Delta_{c} / 2}^{\Delta_{c} / 2} \int_{-\Delta_{c} / 2}^{\Delta_{c} / 2} Q \mathrm{~d} x_{1} \mathrm{~d} x_{2} \mathrm{~d} x_{3} .
$$

Applying (A 1) to both scalar fields $\psi$ and $T$, the volume average of $T \psi$ is

$$
\begin{aligned}
\widetilde{T \psi} & =\frac{1}{\Delta_{c}^{3}} \int_{-\Delta_{c} / 2}^{\Delta_{c} / 2} \int_{-\Delta_{c} / 2}^{\Delta_{c} / 2} \int_{-\Delta_{c} / 2}^{\Delta_{c} / 2} T \psi \mathrm{d} x_{1} \mathrm{~d} x_{2} \mathrm{~d} x_{3} \\
& =T_{0} \psi_{0}+\frac{\Delta_{c}^{2}}{12} \frac{\partial \widetilde{T}}{\partial x_{i}} \frac{\partial \widetilde{\psi}}{\partial x_{i}}+T^{\prime \prime} \frac{\widetilde{\partial \widetilde{\psi}}}{\partial x_{j}} x_{j}+\psi^{\prime \prime} \frac{\widetilde{\partial \widetilde{T}}}{\partial x_{j}} x_{j}+\widetilde{T^{\prime \prime} \psi^{\prime \prime}} .
\end{aligned}
$$

Observing that $\widetilde{\psi}=\psi_{0}$ (and similarly $\widetilde{T}=T_{0}$ ), and neglecting the last three terms of the latter expression, since these are associated with fluctuations,

$$
\widetilde{T \psi}-\widetilde{T} \widetilde{\psi} \simeq \frac{\Delta_{c}^{2}}{12} \frac{\partial \widetilde{T}}{\partial x_{i}} \frac{\partial \widetilde{\psi}}{\partial x_{i}} .
$$

While the subgrid scalar flux (models (3.19b) and (3.19d)) relies on the subgrid vortex motion through the subgrid kinetic energy $\widetilde{k}$ (which can be linked to the local angular velocity, see Pullin 2000), the model (3.19e) based on (A 4) is independent of the way the vortex winds each scalar field $\psi$ and $T$. However, considering the high-order terms in (A $3 b$ ) would possibly introduce a dependence on the subgrid vortical flow. A result similar to (A 4) was obtained by Moeleker \& Leonard (2001), who used a deconvolution approach with a specific spatial (Gaussian) filter. Their subgrid-scale model is based on a truncated exact series expansion for Gaussian-filtered products. Retaining only the first two terms in the series,

$$
\widetilde{T \psi}-\widetilde{T} \widetilde{\psi} \simeq \frac{\sigma^{2}}{2} \frac{\partial \widetilde{T}}{\partial x_{i}} \frac{\partial \widetilde{\psi}}{\partial x_{i}},
$$

where $\sigma$ is the filter's characteristic length scale. The models (A 4) and (A 5) are identical with the choice $\sigma=\Delta_{c} / \sqrt{6}$. 

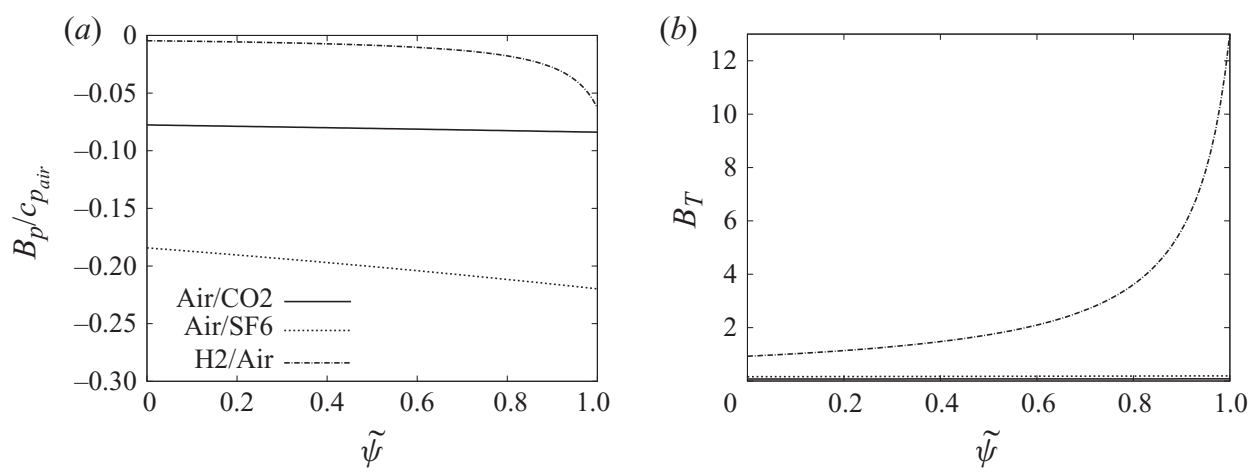

FIGURE 17. Dimensionless pressure and temperature subgrid factors $(a) B_{p} / c_{p_{a i r}}$ and $(b) B_{T}$ versus $\widetilde{\psi}$ for the three gas combinations air $/ \mathrm{CO}_{2}$, air $/ \mathrm{SF}_{6}$ and air $/ \mathrm{H}_{2}$.

If the cutoff scale is within the inertial subrange, the magnitude of $\widetilde{T \psi}-\widetilde{T} \widetilde{\psi}$ is expected to scale as

$$
|\widetilde{T \psi}-\widetilde{T} \widetilde{\psi}| \sim\left(\frac{\Delta_{c}}{L_{\varepsilon}}\right)^{2 / 3}{\widetilde{T^{\prime \prime 2}}}^{1 / 2}{\widetilde{\psi^{\prime \prime 2}}}^{1 / 2}
$$

where $L_{\varepsilon}=u^{\prime 3} / \varepsilon$ defines a dissipation length, with $u^{\prime}$ being the r.m.s. velocity, and where we recall that $\varepsilon$ represents the dissipation rate of kinetic energy. To obtain this result, we start from (A 4):

$$
|\widetilde{T \psi}-\widetilde{T} \widetilde{\psi}| \sim \Delta_{c}^{2} \frac{\delta \widetilde{T}}{\Delta_{c}} \frac{\delta \widetilde{\psi}}{\Delta_{c}} .
$$

We then assume a scalar spectrum of the Obukov-Corrsin form (inertial subrange)

$$
E_{\psi}(k)=\beta \varepsilon^{-1 / 3} \varepsilon_{\psi} k^{-5 / 3}, \quad k \eta_{B}<1,
$$

where $\beta$ is a universal constant, $\varepsilon_{\psi}$ is the dissipation rate of scalar variance, and $\eta_{B}=\left(v^{3} / \varepsilon\right)^{1 / 4} S^{-1}$ is the Batchelor scale. The local changes $\delta \widetilde{T}$ and $\delta \widetilde{\psi}$ can be related to their respective spectra through a second-order scalar structure function matching at separation $\Delta_{c}$. For example, for $\psi$,

$$
(\delta \widetilde{\psi})^{2} \sim \varepsilon^{-1 / 3} \varepsilon_{\psi} \Delta_{c}^{2 / 3} .
$$

Using $\varepsilon_{\psi} \sim \widetilde{\psi^{\prime \prime 2}} / \tau_{\varepsilon}$, with $\tau_{\varepsilon}=L_{\varepsilon} / u^{\prime}$, we obtain

$$
\delta \widetilde{\psi} \sim\left(\frac{\Delta_{c}}{L_{\varepsilon}}\right)^{1 / 3}{\widetilde{\psi^{\prime \prime 2}}}^{1 / 2} .
$$

Applying the same scaling argument to $\delta \widetilde{T}$ then gives (A 6).

The correlation $\widehat{T \psi}-\widetilde{T} \widetilde{\psi}$ present in the pressure and temperature evaluations (3.16) is multiplied by a factor depending on $m_{\alpha}$ and $c_{p_{\alpha}}(\alpha=1$ and 2), as well as $\widetilde{\psi}$ 
through $\widetilde{c}_{p}$ and $\tilde{m}$ :

$$
\left.\begin{array}{r}
\bar{p}=(\widetilde{\gamma}-1)\left[\bar{E}-\frac{1}{2} \bar{\rho} \widetilde{u}_{i} \widetilde{u}_{i}-\frac{1}{2} \tau_{i i}\right]+B_{p} \bar{\rho}(\widetilde{T \psi}-\widetilde{T} \widetilde{\psi}), \\
B_{p}=\widetilde{c}_{p}(\widetilde{\gamma}-1)\left[\widetilde{m}\left(\frac{1}{m_{1}}-\frac{1}{m_{2}}\right)-\frac{c_{p_{1}}-c_{p_{2}}}{\widetilde{c}_{p}}\right], \\
\widetilde{T}=\frac{\widetilde{\gamma}}{\bar{\rho} \widetilde{c}_{p}}\left(\bar{E}-\frac{1}{2} \widetilde{\rho}_{i} \widetilde{u}_{i}-\frac{1}{2} \tau_{i i}\right)+B_{T}(\widetilde{T \psi}-\widetilde{T} \widetilde{\psi}), \\
B_{T}=(\widetilde{\gamma}-1) \tilde{m}\left(\frac{1}{m_{1}}-\frac{1}{m_{2}}\right)-\widetilde{\gamma} \frac{c_{p_{1}}-c_{p_{2}}}{\widetilde{c}_{p}} .
\end{array}\right\}
$$

From (A 6), the pressure and temperature subgrid corrections have a ratio to the background pressure and temperature fields $\sim B_{p} / c_{p_{\text {ref }}}\left(\Delta_{c} / L_{\varepsilon}\right)^{2 / 3}$ and $\sim B_{T}\left(\Delta_{c} / L_{\varepsilon}\right)^{2 / 3}$ respectively, where $\left(\Delta_{c} / L_{\varepsilon}\right)^{2 / 3}$ is in the range of $0.05-0.5$ (depending on the gas combination considered), based on rough estimates of the plane-averaged TKE and turbulent dissipation computed within the mixing layer. Investigating further, we consider the influence of the gas combination and focus especially on the factors $B_{p}$ and $B_{T}$. From figure 17 (where $c_{p_{\text {ref }}}$ is taken to be $c_{p_{a i r}}$ ), we can see that for the combination air- $\mathrm{H}_{2}$ or $\mathrm{H}_{2}-$ air, $B_{T}$ is about 10-100 times larger than for the other cases. Focusing on high-Atwood ratio magnitudes as cases with potentially important subgrid corrections, we take the limit $A \rightarrow 1^{-}$and assume without loss of generality that $A>0$ (i.e. $m_{1} \gg m_{2}$ ). As the molar heat capacity at constant pressure is given by $C_{p_{\alpha}}=c_{p_{\alpha}} m_{\alpha}, B_{T}=1 /(1-\widetilde{\psi})$ at dominant order. Therefore, $B_{T}$ can be large as $\widetilde{\psi} \rightarrow 1^{-}$, but the effect on the final temperature correction, $B_{T}(\widetilde{T \psi}-\widetilde{T} \widetilde{\psi})$, is compensated for by the fact that the correlation modelled by (A 4) vanishes in the pure heavy-fluid region where spatial gradients of $\widetilde{\psi}$ are zero. Computations actually show that the subgrid pressure/temperature corrections are extremely small compared with the background pressure/temperature fields, and are restricted to the extent of the mixing layer where gradients of $\widetilde{\psi}$ are non-zero, as can be inferred from figure 18 . It is also interesting to observe that, at any given $A$ and for combinations of gases with identical $C_{p_{\alpha}}$ (e.g. pairs of monoatomic or diatomic gases), $B_{p}=0$ and $B_{T}=\left[m_{1} /\left(m_{1}-m_{2}\right)-\widetilde{\psi}\right]^{-1}$, which cannot be large since $\widetilde{\psi} \in[0,1]$ and $\left.m_{1} /\left(m_{1}-m_{2}\right) \notin\right] 0,1[$, except when both limits $m_{1} \gg m_{2}$ and $\widetilde{\psi} \rightarrow 1^{-}$are met. To summarize, in non-reacting flows, at the Mach numbers and gas pairings considered here, subgrid corrections to $\bar{p}$ and $\widetilde{T}$ based on multispecies effects do not play an important role in the mixing-layer growth and pressure/temperature calculations. These corrections, however, could be larger in reacting flows (sharper temperature gradients near reaction zones, correlations of $Y_{\alpha}$ with the formation enthalpies, etc.) and/or in highly energetic flows (where stronger shock waves generate greater anisotropy), and should be, in general, considered for the computations of compressible turbulent mixing in multicomponent flows.

The model (A 4) can also be applied to the correlation $\widetilde{T \partial_{j} \psi}-\widetilde{T} \partial_{j} \widetilde{\psi}$ :

$$
T \widetilde{\frac{\partial \psi}{\partial x_{j}}}-\widetilde{T} \frac{\partial \widetilde{\psi}}{\partial x_{j}}=\frac{\Delta_{c}^{2}}{12} \frac{\partial \widetilde{T}}{\partial x_{i}} \frac{\partial^{2} \widetilde{\psi}}{\partial x_{i} \partial x_{j}},
$$



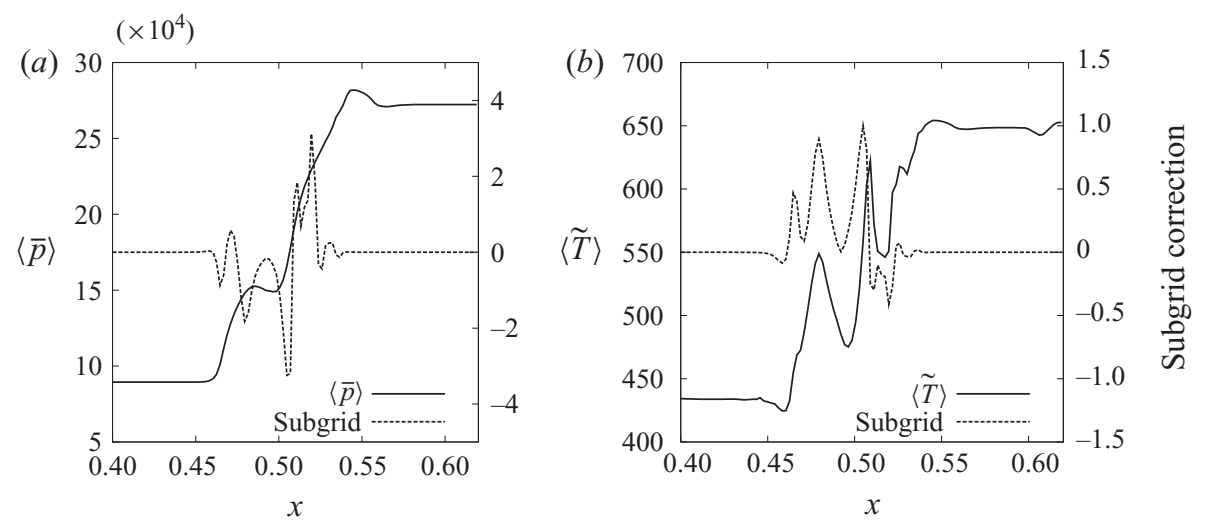

Figure 18. $(a, b)$ Plane-averaged pressure and temperature $\langle\bar{p}\rangle$ and $\langle\widetilde{T}\rangle$, and their respective subgrid corrections $\left\langle B_{p} \bar{\rho}(\widetilde{T \psi}-\widetilde{T} \widetilde{\psi})\right\rangle$ and $\left.\left\langle B_{T}(\widetilde{T \psi}-\widetilde{T} \widetilde{\psi})\right\rangle\right)$. The case shown is $H_{2}$-air, around the reshock time.

and using the same scaling arguments,

$$
\left|\widetilde{T \frac{\partial \psi}{\partial x_{j}}}-\widetilde{T} \frac{\partial \widetilde{\psi}}{\partial x_{j}}\right| \sim \frac{1}{\Delta_{c}}\left(\frac{\Delta_{c}}{L_{\varepsilon}}\right)^{2 / 3}{\widetilde{T^{\prime \prime 2}}}^{1 / 2}{\widetilde{\psi^{\prime \prime 2}}}^{1 / 2} .
$$

As a result, the ratio of the subgrid interdiffusional enthalpy flux $q_{j}^{T \partial \psi}$ (see (3.19c)) to the turbulent temperature flux $q_{j}^{T}$ (see $(3.19 b)$ ) is $\sim\left(L_{\varepsilon} / \Delta_{c}\right)^{2 / 3} /(\operatorname{Re} S c) \lesssim 10^{-2}$, since the Reynolds number $R e=u^{\prime} L_{\varepsilon} / v_{\text {ref }}$ is a typically large number (estimated here in the range of $10^{3}-10^{5}$ ) and the Schmidt number $S c=v_{\text {ref }} / D_{\text {ref }}$ of the order or greater than unity for the mixtures considered. Similarly, the resolved interdiffusional enthalpy flux $\breve{q}_{d_{j}}($ see $(3.14 c))$ is negligible compared to $q_{j}^{T}$ as their ratio scales like $\left(L_{\varepsilon} / \Delta_{c}\right) /(\operatorname{Re} S c)$.

\section{REFERENCES}

Alon, U., Hecht, J., Ofer, D. \& Shvarts, D. 1995 Power laws and similarity of Rayleigh-Taylor and Richtmyer-Meshkov mixing fronts at all density ratios. Phys. Rev. Lett. 74 (4), 534-537.

Andronov, V. A., Bakhrakh, S. M., Meshrov, E. E., Mokhov, V. N., Nikiforov, V. V., Pevnitskit, A. V. \& Tolshmyakov, A. I. 1976 Turbulent mixing at contact surface accelerated by shock waves. Sov. Phys. JETP 44 (2), 424-427.

Arnett, D., Bahcall, J. N., Kirshner, R. P. \& Woosler, S. E. 1989 Supernova 1987A. Annu. Rev. Astron. Astrophys. 27, 629-700.

Berger, M. J. \& Colella, P. 1989 Local adaptive mesh refinement for shock hydrodynamics. J. Comput. Phys. 82 (1), 64-84.

Berger, M. J. \& Oliger, J. 1984 Adaptive mesh refinement for hyperbolic partial-differential equations. J. Comput. Phys. 53 (3), 484-512.

Blaisdell, G. A. 1991 Numerical simulation of compressible homogeneous turbulence. PhD thesis, Stanford University.

Bond, C., Hill, D. J., Meiron, D. I. \& Dimotakis, P. E. 2009 Shock structure in a planar convergent geometry with experiment and simulation. J. Fluid Mech. 641, 297-333.

Brouillette, M. \& Sturtevant, B. 1989 Growth induced by multiple shock waves normally incident on plane gaseous interfaces. Physica D 37, 248-263.

Brouillette, M. \& Sturtevant, B. 1993 Experiments on the Richtmyer-Meshkov instability: small-scale perturbations on a plane interface. Phys. Fluids A 5 (4), 916-930.

Brouillette, M. \& Sturtevant, B. 1994 Experiments on the Richtmyer-Meshkov instability: single-scale perturbations on a continuous interface. J. Fluid Mech. 263, 271-292. 
Burrows, A., Hayes, J. \& FryXell, B. A. 1995 On the nature of core-collapse supernova explosions. Astrophys. J. 450, 830-850.

Canuto, V. M. \& Goldman, I. 1985 Analytical model for large-scale turbulence. Phys. Rev. Lett. 54 (5), 430-433.

CharakhChyan, A. A. 2001 Reshocking at the non-linear stage of Richtmyer-Meshkov instability. Plasma Phys. Control. Fusion 43, 1169-1179.

Collins, B. D. \& JACOBS, J. W. 2002 PLIF flow visualization and measurements of the RichtmyerMeshkov instability of an air/SF 6 interface. J. Fluid Mech. 464, 113-136.

DeITERDing, R. 2005 Construction and application of an AMR algorithm for distributed memory computers. In Adaptive Mesh Refinement: Theory and Applications (ed. T. Plewa, T. Linde \& V. G. Weirs), pp. 361-372. Lecture Notes in Computational Science and Engineering, vol. 41. Springer.

Deiterding, R., Radovitzky, R., Mauch, S. P., Noels, L., Cummings, J. C. \& Meiron, D. I. 2006 A virtual test facility for the efficient simulation of solid material response under strong shock and detonation wave loading. Engng Comput. 22 (3-4), 325-347.

Dimonte, G. 2004 Dependence of turbulent Rayleigh-Taylor instability on initial perturbations. Phys. Rev. E 69, 056305 (1-14).

Dimonte, G., Ramaprabhu, P. \& Andrews, M. 2007 Rayleigh-Taylor instability with complex acceleration history. Phys. Rev. E 76, 046313 (1-6).

Dimonte, G. \& Schneider, M. 2000 Density ratio dependence of Rayleigh-Taylor mixing for sustained and impulsive acceleration histories. Phys. Fluids 12 (2), 304-321.

Dimotakis, P. E. 2000 The mixing transition in turbulent flows. J. Fluid Mech. 409, 69-97.

DufF, R. E., Harlow, F. H. \& HiRT, C. W. 1962 Effects of diffusion on interface instability between gases. Phys. Fluids 5, 417-425.

Erez, L., Sadot, O., Oron, D., Erez, G., Levin, L. A., Shvarts, D. \& Ben-Dor, G. 2000 Study of the membrane effect on turbulent mixing measurements in shock tubes. Shock Waves 10 (4), 241-251.

Ghosal, S. 1996 An analysis of numerical errors in large-eddy simulations of turbulence. J. Comput. Phys. 125 (1), 187-206.

Ghosal, S. 1999 Mathematical and physical constraints on large-eddy simulation of turbulence. AIAA J. 37 (4), 425-433.

Giordano, J. \& Burtschell, Y. 2006 Richtmyer-Meshkov instability induced by shock-bubble interaction: numerical and analytical studies with experimental validation. Phys. Fluids 18 (3), 036102.

Gottlieb, S., Shu, C.-W. \& Tadmor, E. 2001 Strong stability-preserving high-order time discretization methods. SIAM Rev. 43 (1), 89-112.

Greenough, J. A. \& Burke, E. 2004 The effect of initial conditions on late time asymptotics and mixing for multimode Richtmyer-Meshov instability. In Ninth International Workshop on the Physics of Compressible Turbulent Mixing, 19-23 July, Cambridge, UK.

Gupta, M. R., Roy, S., Sarkar, S., Khan, M., Pant, H. C. \& Srivastava, M. K. 2007 Effect on Richtmyer-Meshkov instability of deviation from sinusoidality of the corrugated interface between two fluids. Laser Part. Beams 25, 503-510.

Gutmark, E. \& Wygnanski, I. 1976 The planar turbulent jet. J. Fluid Mech. 73 (3), 465-495.

Hill, D. J., Pantano, C. \& Pullin, D. I. 2006 Large-eddy simulation and multi-scale modeling of Richtmyer-Meshkov instability with reshock. J. Fluid Mech. 557, $29-61$.

Hill, D. J. \& Pullin, D. I. 2004 Hybrid tuned center-difference-WENO method for large eddy simulations in the presence of strong shocks. J. Comput. Phys. 194 (2), 435-450.

Holmes, R. L., Dimonte, G., Fryxell, B., Gittings, M. L., Grove, J. W., Schneider, M., Sharp, D. H., Velikovich, A. L., Weaver, R. P. \& Zhang, Q. 1999 Richtmyer-Meshkov instability growth: experiment, simulation and theory. J. Fluid Mech. 389, 55-79.

Honern, A. E. \& Morn, P. 2004 Higher entropy conservation and numerical stability of compressible turbulence simulations. J. Comput. Phys. 201 (2), 531-545.

Houas, L. \& Chemouni, I. 1996 Experimental investigation of Richtmyer-Meshkov instability in shock tube. Phys. Fluids 8 (2), 614-627.

Kosovic, B., Pullin, D. I. \& Samtaney, R. 2002 Subgrid-scale modeling for large-eddy simulations of compressible turbulence. Phys. Fluids 14 (4), 1511-1522. 
Latini, M., Schilling, O. \& Don, W. S. 2007 Effects of WENO flux reconstruction order and spatial resolution on reshocked two-dimensional Richtmyer-Meshkov instability. J. Comput. Phys. 221 (2), 805-836.

LaYes, G., Jourdan, G. \& Houas, L. 2009 Experimental study on a plane shock wave accelerating a gas bubble. Phys. Fluids 21 (7), 074102.

Leinov, E., Malamud, G., Elbaz, Y., Levin, L. A., Ben-Dor, G., Shvarts, D. \& Sadot, O. 2009 Experimental and numerical investigation of the Richtmyer-Meshkov instability under re-shock conditions. J. Fluid Mech. 626, 449-475.

Lindl, J. D., McCrory, R. L. \& CAmpbell, E. M. 1992 Progress towards ignition and burn propagation in inertial confinement fusion. Phys. Today 45, 32-40.

Lombardini, M. 2008 Richtmyer-Meshkov instability in converging geometries. PhD thesis, Caltech.

Lombardini, M., Deiterding, R. \& Pullin, D. I. 2008 Large-eddy simulation of the RichtmyerMeshkov instability in a converging geometry. In Quality and Reliability of Large-Eddy Simulations, Proc. of QLES 2007 Intl Symposium (ed. J. Meyers, B. J. Geurts \& P. Sagaut), ERCOFTAC Series, vol. 12, pp. 23-51. Springer.

Lombardini, M. \& Pullin, D. I. 2009 Startup process in the Richtmyer-Meshkov instability. Phys. Fluids 21 (4), 044104.

LundGren, T. S. 1982 Strained spiral vortex model for turbulence fine structure. Phys. Fluids 25 (12), 2193-2203.

Meshkov, E. E. 1969 Instability of the interface of two gases accelerated by a shock wave. Sov. Fluid Dyn. 4 (5), 101-108.

Mikaelian, K. O. 1989 Turbulent mixing generated by Rayleigh-Taylor and Richtmyer-Meshkov instabilities. Physica D 36, 343-347.

Mikaelian, K. O. 1991 Density gradient stabilization of the Richtmyer-Meshkov instability. Phys. Fluids A 3 (11), 2638-2643.

Mikaelian, K. O. 2009 Reshocks, rarefactions, and the generalized Layzer model for hydrodynamic instabilities. Phys. Fluids 21 (2), 024103.

Misra, A. \& Pullin, D. I. 1997 A vortex-based model for large-eddy simulation. Phys. Fluids 9 (8), 2443-2454.

Moeleker, P. \& Leonard, A. 2001 Lagrangian methods for the tensor-diffusivity subgrid model. J. Comput. Phys. 167 (1), 1-21.

Motl, B., Oakley, J., Ranjan, D., Weber, C., Anderson, M. \& Bonazza, R. 2009 Experimental validation of a Richtmyer-Meshkov scaling law over large density ratio and shock strength ranges. Phys. Fluids 21 (12), 126102.

Niederhaus, J. H. J., Greenough, J. A., Oakley, J. G., Ranjan, D., Anderson, M. H. \& BonAzZA, R. 2008 A computational parameter study for the three-dimensional shock-bubble interaction. J. Fluid Mech. 594, 85-124.

Orlicz, G. C., Balakumar, B. J., Tomkins, C. D. \& Prestridge, K. P. 2009 A Mach number study of the Richtmyer-Meshkov instability in a varicose, heavy-gas curtain. Phys. Fluids 21 (6), 064102.

Oron, D., Arazi, L., Kartoon, D., Rikanati, A., Alon, U. \& Shvarts, D. 2001 Dimensionality dependence of the Rayleigh-Taylor and Richtmyer-Meshkov instability late-time scaling laws. Phys. Plasmas 8 (6), 2883-2889.

Pantano, C., Deiterding, R., Hill, D. J. \& Pullin, D. I. 2007 A low numerical dissipation patchbased adaptive mesh refinement method for large-eddy simulation of compressible flows. J. Comput. Phys. 221 (1), 63-87.

Pullin, D. I. 2000 A vortex-based model for the subgrid flux of a passive scalar. Phys. Fluids 12 (9), 2311-2319.

READ, K. I. 1984 Experimental investigation of turbulent mixing by Rayleigh-Taylor instability. Physica D 12, 45-58.

Reid, R. C., Prausnitz, J. M. \& Polling, B. E. 1987 The Properties of Gases and Liquids. McGrawHill.

Richtmyer, R. D. 1960 Taylor instability in shock acceleration of compressible fluids. Commun. Pure Appl. Maths. 13, 297-319.

Saffman, P. G. \& Meiron, D. I. 1989 Kinetic energy generated by the incompressible RichtmyerMeshkov instability in a continuously stratified fluid. Phys. Fluids A 1 (11), 1767-1771. 
SAmtaney, R. \& Zabusky, N. J. 1993 Circulation deposition on shock-accelerated planar and curved density-stratified interfaces: models and scaling laws. J. Fluid Mech. 269, 45-78.

Schilling, O., Latini, M. \& Don, W. S. 2007 Physics of reshock and mixing in single-mode Richtmyer-Meshkov instability. Phys. Rev. E 76, 026319.

Taccetti, J. M., Batha, S. H., Fincke, J. R., Delamater, N. D., Lanier, N. E., Magelssen, G. R., Hueckstaedt, R. M., Rothman, S. D., Horsfield, C. J. \& Parker, K. W. 2005 RichtmyerMeshkov instability reshock experiments using laser-driven double-cylinder implosions. Astrophys. Space Sci. 298, 327-331.

Tomkins, C., Kumar, S., Orlicz, G. \& Prestridge, K. 2008 An experimental investigation of mixing mechanisms in shock-accelerated flow. J. Fluid Mech. 611, 131-150.

Vetter, M. \& Sturtevant, B. 1995 Experiments on the Richtmyer-Meshkov instability of an air/SF 6 interface. Shock Waves 4, 247-252.

Voelkl, T. \& Pullin, D. I. 2000 A physical-space version of the stretched-vortex subgrid-stress model for large-eddy simulations. Phys. Fluids 12 (7), 1810-1825.

Youngs, D. L. 1984 Numerical simulation of turbulent mixing by Rayleigh-Taylor instability. Physica D 12, 32-44.

ZABUSKY, N. J. 1999 Vortex paradigm for accelerated inhomogeneous flows: visiometrics for the Rayleigh-Taylor and Richtmyer-Meshkov environments. Annu. Rev. Fluid Mech. 31, 495-536.

Zaitsev, S. V., Lazareva, E. V., Chernukha, V. V. \& Belyaev, V. M. 1985 An experimental investigation and numerical modeling of turbulent mixing in one-dimensional flows. Sov. Phys. Dokl. 30, 579.

Zhang, T. A., Dahlburg, R. B. \& Dahlburg, J. P. 1992 Direct and large-eddy simulations of three-dimensional compressible Navier-Stokes turbulence. Phys. Fluids A 4, 127-140. 